\title{
The impact of the SZ effect on cm-wavelength (1-30 GHz) observations of galaxy cluster radio relics
}

\author{
Kaustuv Basu ${ }^{1}$, Franco Vazza ${ }^{2}$, Jens Erler ${ }^{1}$, and Martin Sommer ${ }^{1}$ \\ 1 Argelander Institut für Astronomie, Universität Bonn, Auf dem Hügel 71, 53121 Bonn, Germany \\ e-mail: kbasu@astro.uni-bonn.de \\ 2 Hamburger Sternwarte, Gojenbergsweg 112, 21029 Hamburg, Germany
}

Received 10 November 2015 / Accepted 1 April 2016

\begin{abstract}
Radio relics in galaxy clusters are believed to be associated with powerful shock fronts that originate during cluster mergers, and are a testbed for the acceleration of relativistic particles in the intracluster medium. Recently, radio relic observations have pushed into the $\mathrm{cm}$-wavelength domain $(1-30 \mathrm{GHz})$ where a break from the standard synchrotron power law spectrum has been found, most noticeably in the famous "Sausage" relic. Such spectral steepening is seen as an evidence for non-standard relic models, such as ones requiring seed electron population with a break in their energy spectrum. In this paper, however, we point to an important effect that has been ignored or considered insignificant while interpreting these new high-frequency radio data, namely the contamination due to the Sunyaev-Zel'dovich (SZ) effect that changes the observed synchrotron flux. Even though the radio relics reside in the cluster outskirts, the shock-driven pressure boost increases the SZ signal locally by roughly an order of magnitude. The resulting flux contamination for some well-known relics are non-negligible already at $10 \mathrm{GHz}$, and at $30 \mathrm{GHz}$ the observed synchrotron fluxes can be diminished by a factor of several from their true values. At higher redshift the contamination gets stronger due to the redshift independence of the SZ effect. Interferometric observations are not immune to this contamination, since the change in the SZ signal occurs roughly at the same length scale as the synchrotron emission, although there the flux loss is less severe than single-dish observations. Besides presenting this warning to observers, we suggest that the negative contribution from the SZ effect can be regarded as one of the best evidence for the physical association between radio relics and shock waves. We present a simple analytical approximation for the synchrotron-to-SZ flux ratio, based on a theoretical radio relic model that connects the nonthermal emission to the thermal gas properties, and show that by measuring this ratio one can potentially estimate the relic magnetic fields or the particle acceleration efficiency.
\end{abstract}

Key words. galaxies: clusters: intracluster medium - shock waves - radiation mechanisms: non-thermal radiation mechanisms: thermal

\section{Introduction}

Along with their cosmological relevance, galaxy clusters are giant laboratories for studying many interesting astrophysical processes. Often, different astrophysical phenomena, which are observed in completely different regimes of the electromagnetic spectrum, are connected by the same underlying cause. A prominent example of such a case is cluster merger shocks. Shocks cause a jump in the density and temperature of the thermal intracluster medium (ICM), and are believed to produce nonthermal cosmic ray electrons (CRe) through the first-order Fermi acceleration. The former effect can be observed in the soft X-ray band or in the millimeter/submillimeter wavebands through the SunyaevZel'dovich effect (thermal SZ effect, tSZ effect, or simply SZ for short; Sunyaev \& Zel'dovich 1972, 1980), whereas the latter is visible in the radio wavebands through synchrotron emission or in the hard X-rays through inverse Compton emission. This paper aims to explore some observational consequences of when the radio synchrotron signal and the SZ effect overlap each other at $\mathrm{cm}$ wavelengths $(1-30 \mathrm{GHz})$.

Clusters grow through mergers and these mergers drive shock waves through the ICM (e.g., Sarazin 2002). In cluster simulations shocks are ubiquitous (e.g., Nuza et al. 2012; Skillman et al. 2013; Hong et al. 2014), but their direct observation is still limited by small number statistics as detecting
ICM shocks are hampered by their low surface brightness and projection effects, particularly in the cluster outskirts where the thermal signature is hard to detect in SZ or X-rays (e.g., Akamatsu \& Kawahara 2013; Planck Collaboration X 2013; Erler et al. 2015). Radio relics are elongated, diffuse synchrotron sources found in the cluster outskirts, have scale lengths on the order of $\sim 1 \mathrm{Mpc}$ and are not associated with any AGN outflow (e.g., review by Feretti et al. 2012). The common wisdom is that these radio relics mark the location of powerful shock fronts generated by cluster mergers, which were first postulated by Enßlin et al. (1998). The mechanism behind the acceleration of GeV energy electrons at the shock fronts is believed to be diffusive shock acceleration (DSA; Blandford \& Eichler 1987), and have been the subject of extensive studies both analytically and through numerical simulations (e.g., Hoeft \& Brüggen 2007; Pfrommer et al. 2008; Nuza et al. 2012; Kang et al. 2012; Skillman et al. 2013; Pinzke et al. 2013; Vazza \& Brüggen 2014; Vazza et al. 2015). However, the problem with the DSA-based scenario is the low Mach number of merger shocks inside a cluster volume ( $\mathcal{M} \lesssim 4$; Ryu et al. 2003; Pfrommer et al. 2006), which would make the acceleration efficiency prohibitively low. To overcome this problem special modification to the ambient MaxwellJütner distribution of thermal electrons have been proposed, for example, in the form of a $\kappa$-distribution to add a suprathermal tail (e.g., Kang et al. 2014), or by postulating a pre-existing 
population of $\mathrm{CRe}$ from past AGN activities at the cluster core (e.g., Pinzke et al. 2013). On the other hand, recent particle-incell simulations have suggested alternative mechanisms based on shock drift acceleration (SDA) that can efficiently inject thermal electrons into a DSA-like cycle, thus eliminating any need for an additional nonthermal population beforehand (Matsukiyo et al. 2011; Guo et al. 2014b,a). Thus it is of utmost importance to find observational signatures of the underlying CRe energy distribution that causes radio relics to find out whether a seed population of $\mathrm{CRe}$ exists or not prior to the shock passage.

One way of knowing the particle injection spectrum and the associated magnetic field in the relics is to measure the relic fluxes out to high frequencies, looking for spectral break in the relic spectrum that can indicate deviation from a power law for the seed CRe population. Recently, some spectacular examples of a spectral break in relic spectrum have been published in the literature, in particular, for the famous Sausage relic in the cluster CIZA J2242.8+5301 (Stroe et al. 2014, 2016). Based on the radio interferometric data obtained at $16 \mathrm{GHz}$, Stroe et al. (2014) first found a pronounced steepening of the integrated relic spectrum at high frequencies, which is inconsistent with the extrapolation of the low-frequency spectrum at $12 \sigma$. Recent results for the Sausage and Toothbrush relics published by Stroe et al. (2016) extends the spectral measurements out to $30 \mathrm{GHz}$, with a consistent trend for spectral steepening. Such steepening at high frequencies is not expected according to the standard DSA-based acceleration scenario, since there is a balance between particle injection and ageing, hence these recent observations have been used as an argument for the presence of aged fossil electrons or strong magnetic field downstream (Stroe et al. 2016). Another famous relic that was observed out to $10 \mathrm{GHz}$ is in the cluster A2256 (Trasatti et al. 2015) and also shows mild evidence of spectral steepening.

In light of these new results, a careful investigation of the amplitude of the SZ signal at radio relic location is necessary, a problem that has not been investigated in detail so far. We try to address this issue comprehensively with a semianalytical approach. The SZ effect appears as a negative signal against the CMB background for frequencies below $220 \mathrm{GHz}$. At the $\mathrm{cm}$ wavebands of interest $(1-30 \mathrm{GHz})$ the synchrotron power drops rapidly owing to its steep spectrum, whereas the SZ effect increases in strength as $v^{2}$; furthermore, at the location of relics the SZ signal gets boosted by the shock roughly proportional to the Mach number squared. Since the SZ signal boost occurs within the same spatial scales responsible for the radio relic emission, the decrement is not completely filtered out by interferometric observations. We show that for many relics the flux modification due to the SZ effect is non-negligible; it can reduce the observed synchrotron flux by $\sim 10-50 \%$ at $15 \mathrm{GHz}$ and up to a factor of several at $30 \mathrm{GHz}$. These estimates are mostly for radio relic clusters at $z \lesssim 0.2$, and the contamination increases rapidly with redshift. In fact, far from being a simple "nuisance" for observations, the SZ decrement at this frequency range can probably offer the best evidence for the widely accepted relic-shock connection. Our modeling shows that, within a factor $\sim 2$ or so, the predicted SZ decrement is consistent with the reported highfrequency steepening of relics.

The layout of the paper is as following. In Sect. 2 we briefly describe the theory behind the SZ effect and the synchrotron emission from radio relics, in particular, how these signals are expected to behave across a shock front. We introduce an empirical toy model for the synchrotron emissivity profile, and illustrate the flux contamination issue realistically with simulations for interferometric observation. Section 3 presents the results: first some general characteristics of the SZ flux contamination, for example, how the signals change with cluster mass, redshift, and the shock Mach number, and then individual estimates for the flux contamination in some well-known radio relics. In Sect. 4 we provide an analytical approximation for the radio synchrotron-to-SZ flux ratio, and we discuss how its measurement can help to refine the theory of particle acceleration for radio relic origin or constrain the relic magnetic fields. We summarize our work in Sect. 5, and conclude with some general warnings for observers and suggestions for future theoretical work. For computing the distances and measured fluxes, we assume a standard $\Lambda$ CDM cosmology throughout the paper: $H_{0}=71 \mathrm{~km} \mathrm{~s}^{-1} \mathrm{Mpc}^{-1}, \Omega_{\mathrm{m}}=0.27$ and $\Omega_{\Lambda}=0.73$. The redshift-dependent Hubble ratio is defined as $E(z) \equiv H(z) / H_{0}=$ $\sqrt{(1+z)^{3} \Omega_{\mathrm{m}}+\Omega_{\Lambda}}$.

\section{Theoretical and observational basics}

We describe the basics of the Sunyaev-Zel'dovich (SZ) effect, and explain how the SZ and synchrotron signals are boosted by the passage of a shock. To model the synchrotron flux realistically, we construct an empirical model and compare with published relic emission profiles. We also outline the main differences between interferometric and single-dish imaging techniques, and present simulation results for interferometric observation of radio relics in presence of a non-negligible SZ signal.

\section{1. "Negative flux" from the $S Z$ effect}

The SZ effect at $\mathrm{cm}$ wavelengths acts as a contaminant to the synchrotron flux, making the observed flux less than its true value. This is because at the $\mathrm{cm}$ to $\mathrm{mm}$ wavelengths the $\mathrm{SZ}$ signal appears as a decrement in the background CMB radiation intensity. Since all flux measurements are done against the backdrop of the CMB radiation, which defines the "zero level" of flux, for all practicality we can consider the SZ signal a "true" negative signal as opposed to the positive synchrotron flux (for example). In practice, interferometric or single-dish observation sample the distribution of positive and negative fluxes differently, resulting in very different total power images. This we discuss in Sect. 2.5.

The change in the CMB temperature due to the thermal $\mathrm{SZ}$ effect is given by the well-known formula

$\Delta T / T_{\mathrm{CMB}}=f(x) y$,

where $x$ is the dimensionless frequency, $y$ is the Comptonization parameter, $T_{\mathrm{CMB}}=2.726 \mathrm{~K}$, and the frequency spectrum of the temperature variation has the form

$f(x)=\left(x \frac{\mathrm{e}^{x}+1}{\mathrm{e}^{x}-1}-4\right)\left[1+\delta_{\mathrm{rSZ}}\left(x, T_{\mathrm{e}}\right)\right]$.

The second term in parenthesis is the relativistic correction to the SZ effect, and even though the passage of a merger shock is expected to heat up the ICM to $\sim 10 \mathrm{keV}$ or more right behind the shock, this relativistic term can be safely ignored in the frequency range of interest $(\$ 30 \mathrm{GHz})$. In the Rayleigh-Jeans limit $(x \lesssim 0.5)$, we thus obtain $f(x) \approx-2$, and the change in brightness temperature is negative. Converting $\Delta T_{\mathrm{CMB}}$ to Rayleigh-Jeans temperature by multiplying with $x^{2} \mathrm{e}^{x} /\left(\mathrm{e}^{x}-1\right)^{2}$, one can obtain the following simplified expression for the measured negative 
flux density in a given instrument beam (e.g., Birkinshaw 1999):

$\left(\frac{S_{v}}{\mathrm{mJy} / \text { beam }}\right)=\frac{1}{340}\left(\frac{\Delta T_{\mathrm{RJ}}}{\mathrm{mK}}\right)\left(\frac{v}{\mathrm{GHz}}\right)^{2}\left(\frac{\Omega_{\text {beam }}}{\operatorname{arcmin}^{2}}\right)$.

In this formula $\Omega_{\text {beam }}$ is the beam solid angle, relating to the half-power beam width as approximately $\Omega_{\text {beam }}=1.13 \theta_{\text {FWHM }}^{2}$. The factor 1.13 is dropped when we express the SZ flux density independent of telescope beams, in units of mJy/arcmin ${ }^{2}$.

The Comptonization parameter, $y$, is obtained through the line-of-sight integral of the cluster pressure distribution

$y=\frac{\sigma_{\mathrm{T}}}{m_{\mathrm{e}} c^{2}} \int_{\text {l.o.s. }} P_{\mathrm{e}}(r) \mathrm{d} l$.

For the spherical pressure distribution, we assume the Generalized Navarro-Frenk-White, or GNFW, pressure model (Nagai et al. 2007) with the mass dependence and parametrization as given by Arnaud et al. (2010),

$P_{\mathrm{e}}(r)=1.65 \times 10^{-3} E(z)^{8 / 3}\left[\frac{M_{500}}{3 \times 10^{14} M_{\odot}}\right]^{0.79} \mathrm{p}\left(\frac{r c_{500}}{r_{500}}\right) \mathrm{keV} \mathrm{cm}^{-3}$

The term $\mathrm{p}\left(r c_{500} / r_{500}\right)$ gives the "universal" shape of the cluster pressure profile with various slope parameters and the gas concentration parameter $c_{500}$. In the cluster outskirts where radio relics are located, the pressure falls off rapidly, such as $P(r) \propto r^{-5.5}$ or similar, hence a low amplitude of the SZ signal is expected. There is about $30 \%$ scatter in the cluster pressure amplitude (Arnaud et al. 2010; Planck Collaboration V 2013; Sayers et al. 2013), but this is within our accuracy range for predicting the SZ contamination. In Sect. 3.2 we further renormalize these GNFW pressure model results with the SZ measurement of an individual cluster whenever possible to make our flux contamination predictions more accurate.

The above is the standard formulation for the SZ signal in a cluster, which produces a "bowl" of negative flux, whose amplitude near the cluster virial radius is orders of magnitude lower than the peak decrement value at the center. But we are dealing with a shock, and the shock will boost the SZ signal locally. This can be computed from the standard Rankine-Hugoniot condition of pressure jump at the location of a shock,

$\frac{P_{\mathrm{d}}}{P_{\mathrm{u}}}=\frac{2 \gamma \mathcal{M}^{2}-(\gamma-1)}{(\gamma+1)}$,

where $\mathcal{M}$ is the sonic Mach number of the shock, $\gamma=5 / 3$ is the adiabatic index, and we define $P_{\mathrm{d}}$ and $P_{\mathrm{u}}$ as the instantaneous pressure in the downstream (post-shock) and upstream (preshock) regions. We see that the pressure jump scales roughly as $\sim \mathcal{M}^{2}$, hence the boost in the SZ signal is non-negligible. Roughly speaking, this creates a step-function like increase in the SZ flux decrement at the location of the radio relic on top of the GNFW bowl. The sharp change in the SZ signal is also important in the context of interferometric observation, as discussed in Sect. 2.5.

To make a realistic model for the SZ flux variation, we need to assume a shock geometry as well as some cooling length behind the shock. For the former, we assume a standard spherical shock geometry. This is clearly a simplification as simulations show that although shock fronts radiate outward in all direction after a major merger, its geometry is not actually spherical due to the inhomogeneous structure of the ICM (e.g., Miniati 2000).
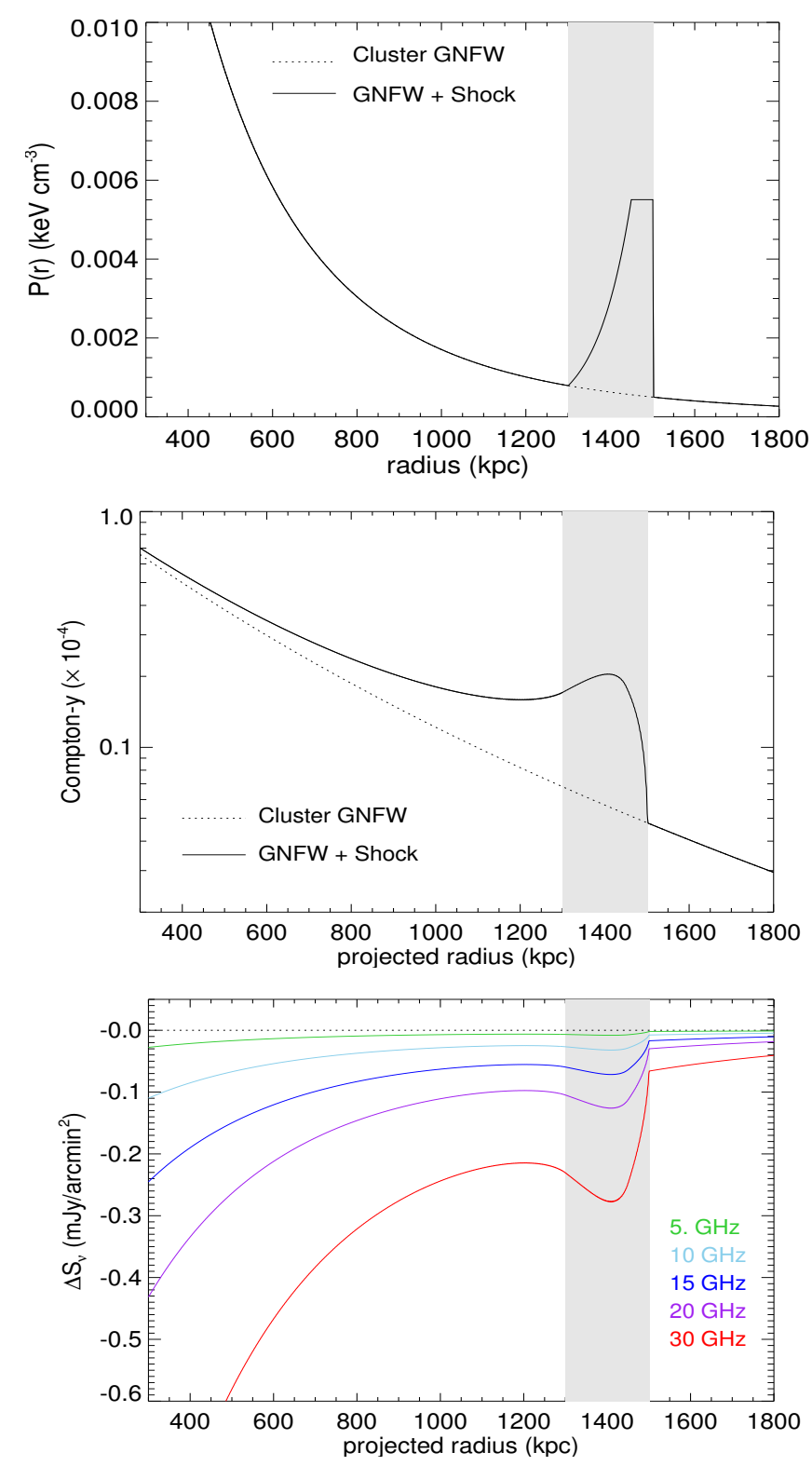

Fig. 1. Illustration of SZ flux decrement at a merger shock, for a spherical shock model at $1.5 \mathrm{Mpc}$ distance from the cluster center, in a $M_{500}=8 \times 10^{14} M_{\odot}$ cluster at $z=0.2$, with shock Mach number $\mathcal{M}=3$. The gray vertical bands indicate the approximate pressureboosted region in radial distance. Top panel: the radial distribution of pressure, demonstrating the localized boost in pressure by roughly factor $\sim 10$. The underlying dotted line is the ambient GNFW pressure model proportional to the mass of the cluster. Middle panel: the projected Compton- $y$ parameter, solid line showing the total value. Bottom panel: the resulting SZ flux densities, in units of $\mathrm{mJy} / \mathrm{arcmin}^{2}$, as would be observed at different frequencies.

However, for our purposes it suffices that the SZ signal boost is localized within the same geometrical boundary as marked by the relics, because the flux contamination is determined primarily from the projection of pressure from a narrow annular section of the shock where the relic resides. The second point is more complicated, namely the width of the shock-boosted region. Lacking any sophisticated simulations for gas cooling, we follow the examples from shock-tube geometry (e.g., Miniati 2007; Vazza et al. 2012), simply assuming that the boosted pressure stays constants for a small distance (which we can call the shock width) after which it drops owing to cooling with a power 
law and merges into the ambient pressure. This setting is shown in the top panel of Fig. 1. We take a small shock width of $50 \mathrm{kpc}$, in line with the most resolved available radio observations (e.g., van Weeren et al. 2010). We ignore any dependence of this width with the shock Mach number. However, we can state that the SZ contamination on radio relic fluxes is not very sensitive to the precise model of the downstream pressure: this is because the radio emission is mostly concentrated just behind the shock front (Sect. 2.2 below), where the pressure closely follows the boosted value according to Eq. (6).

We numerically project this $3 \mathrm{D}$ pressure model to get the Compton- $y$ parameter and the expected flux decrement at any given frequency. The results are shown in Fig. 1 for a fiducial shock model and for a cluster with similar mass and redshift as in the Sausage relic host cluster. The projected distances are expressed in $\mathrm{kpc}$ for ease of comparison with the pressure profile. We see that because of projection the effect of the SZ signal boost extends far inward relative to the shock front, but its amplitude exhibits a local maximum right at the location of the shock width after rising quickly from the ambient SZ signal. In other words, the SZ signal variation is strongest in a relatively narrow region, on the order $\sim 100 \mathrm{kpc}$, where the radio relic is also at its brightest. In our SZ modeling we are neglecting the effect of non-equilibrium between the electrons and ions in the post-shock region. From a simple estimate based on Coulomb interaction time one can expect electron thermalization to happen over a length-scale of $\sim 50 \mathrm{kpc}$ for a $\mathcal{M} \sim 3$ shock, but this represents an upper limit of the effect (Rudd \& Nagai 2009), because it neglects energy exchange mediated by plasma instabilities which can shorten this equilibration timescale significantly (e.g., Bykov et al. 2008). Following from the energy density of the relativistic particles, a nonthermal SZ contribution from the power law electrons should also be very small (Enßlin \& Kaiser 2000).

An important point is that the SZ surface brightness is redshift independent, whereas in contrast, the synchrotron flux rapidly decreases with redshift, both for the redshift dimming and the strong $K$-correction to the observed flux due to the steep synchrotron spectrum of the relics. This implies a rapid increase in the SZ flux contamination for clusters at redshift $z \sim 0.5$ or above, compared to mostly low $-z$ clusters in which radio relics are observed today (Sect. 3.1.1). Not only for radio relics, this redshift dependence of the SZ flux contamination also plays a critical role for $\mathrm{GHz}$-frequency observation of radio halos (Sect. 2.4 below). This is important for the large number of cluster diffuse sources (relics and halos) that are expected to become available from upcoming radio surveys with ASKAP, MeerKAT and SKA.

\subsection{Relic synchrotron emission at the shock front}

In the first-order Fermi process, the momentum distribution of the accelerated particles follow a power law, $f(p) \propto p^{-\delta}$, and the slope of the particle spectrum can be related directly to the shock compression ratio, $r$

$\delta=(r+2) /(r-1)$.

This particle distribution produces synchrotron radiation also with a power law, $S(v) \propto v^{-\alpha_{\text {inj }}}$, where $\alpha_{\text {inj }}=(\delta-1) / 2$ is generally termed as the injection spectrum for the relics. But this is only observable at the very leading edge of the relic, where energy losses are negligible. In practice, the synchrotron spectral index is generally measured over the entire relic area, where particle aging and continuous injection of new particles produce a power law that is steeper, $\alpha_{\text {tot }}=\alpha_{\text {inj }}+0.5$ (Kardashev 1962; Enßlin et al. 1998). We use this integrated spectral index for the rest of our work, simply assuming that the brightness profile of the relic retains its shape at all frequencies. Putting in $\gamma=5 / 3$ in the formula for compression ratio, we get the Mach dependence of the synchrotron spectral index,

$\alpha_{\text {tot }}(=\delta / 2)=\left(\mathcal{M}^{2}+1\right) /\left(\mathcal{M}^{2}-1\right)$.

It can be seen that the spectral index is a weakly varying function of the Mach number for $\mathcal{M} \gtrsim 2$, weak shocks producing a steeper radio spectrum and hence less flux at $\mathrm{cm}$ wavelengths. However, a more pronounced $\mathcal{M}$ dependence can be expected for the amplitude of the radio power at any given frequency, since the kinetic energy dissipated by the shock scales as the third power of shock velocity downstream, hence as $\mathcal{M}^{3}$. This is more pronounced than the roughly $\mathcal{M}^{2}$ increase of the SZ signal due to pressure jump (Eq. (6)). We come back to the $\mathcal{M}$ dependence of the SZ contamination in Sect. 3.1.3 to illustrate the concept qualitatively. More accurate computation of the synchrotron power dependence on the Mach number requires some degree of modeling, because the particle acceleration efficiency and magnetic field amplification by weak cosmological shocks are mostly unknown (e.g., Hoeft \& Brüggen 2007; Pinzke et al. 2013). However, for our work we can avoid these complications using either the measured flux values of known radio relics or some empirical scaling relation between the cluster mass and relic power.

The last piece of information that we need to predict the flux contamination in a realistic manner is the shape of the synchrotron brightness profile. Here, lacking more detailed simulations of CRe production and magnetic fields, we employ an empirical model that matches actual measurements. We find that a lognormal emissivity profile behind the shock front produces acceptable fits to the published relic flux profiles, that can also be modified to fit for the unknown viewing angles of the relics, as described below.

\subsection{A lognormal model for the synchrotron profile}

To make realistic predictions for the flux contamination, we need a synchrotron flux distribution model that can match the observed data at low frequencies as closely as possible and is scalable to the basic relic properties such as its length (largest linear scale, or LLS, of the relic) and the shock Mach number. A crude emissivity model consists of a sharp rise of the synchrotron emissivity at the shock front, followed by a power law decrease downstream as the particles lose energy. We chose a lognormal distribution instead because (i) it makes a more physical change at the shock front that is non-instantaneous and (ii) it offers a simple formulation of the relic width, which we can connect to the particle energy spectrum. This is still a distribution whose tail is flatter than exponential, approximating to a power law beyond the typical width of the shock. The functional form for the flux radial distribution is then

$S_{v}^{\text {sync. }}\left(x_{\mathrm{d}}\right)=S_{0}\left(\frac{\mathrm{e}^{-\left(\log x_{\mathrm{d}}-\mu\right)^{2} / 2 \sigma^{2}}}{\sqrt{2 \pi} x_{\mathrm{d}} \sigma}\right) \quad\left(\mathrm{mJy} / \operatorname{arcmin}^{2}\right)$.

The normalization $S_{0}$ in Eq. (9) above is fixed to match real data (e.g., Sect. 3.2), by integrating the profile above a certain significance level (typically $\sim 20 \sigma$ ) and multiplying by the relic length and mean flux to match total flux values. The two parameters 
$\mathrm{K}$. Basu et al.: SZ contamination in radio relics

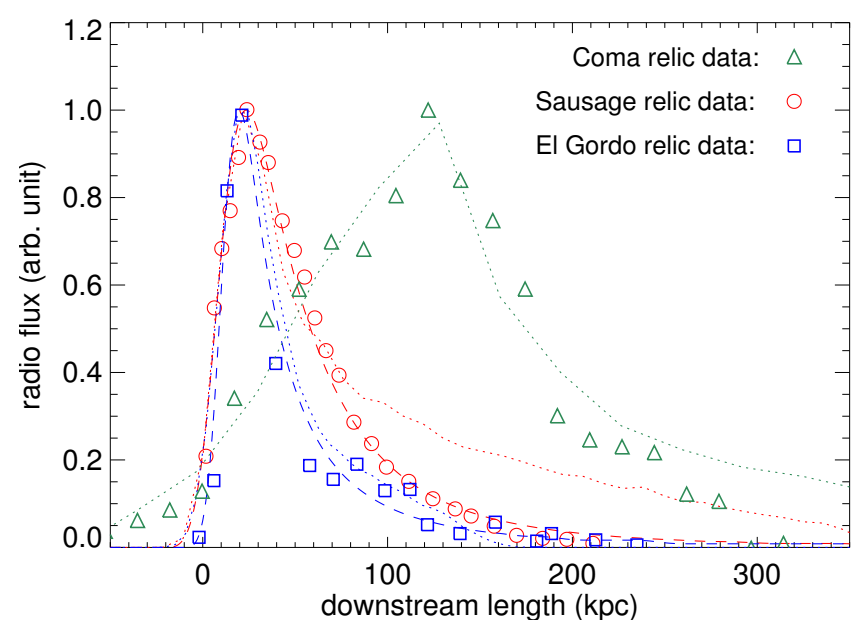

Fig. 2. Fit to the observed flux profiles for three radio relics with our lognormal model. The three different colored symbols represent the flux profile data points in units of physical length, and the dashed and dotted lines with corresponding colors show model fits. The data points are from van Weeren et al. (2010) for the Sausage relic, Lindner et al. (2014) for the El Gordo NW relic, and Trasatti et al. (in prep.) for the Coma relic, all normalized to the same amplitude. For the roughly edge-on relics (Sausage and El Gordo), we show results for two different methods of obtaining a fit: one by directly fitting the projected brightness profile in 1D (dashed lines), and the other using a 3D emissivity model and fitting through projection (dotted lines). The second method produces a long emission tail for the Sausage relic. For the Coma data only a 3D result is shown, which provides an acceptable fit after accounting for $\mathrm{a} \sim 30^{\circ}$ viewing angle (see text). The models are convolved with the respective instrument resolutions as noted in the publications. The shock location is at zero; cluster center is to the right.

$[\mu, \sigma]$ for the shape fitting denote the location and scale of the distribution, and $x_{\mathrm{d}}$ is the downstream length in kpc. There can be two viable methods to obtain the fit: we can either fit the projected emission profile directly using a lognormal model or assume a 3D emissivity profile that follows lognormal distribution and fit the data after projection. We find that at least for edge-on relics both methods can offer reasonable fits to the data, but we prefer the latter method because it allows for a natural way to make a rotation and view the relic from arbitrary angles.

The results for fitting the lognormal model of Eq. (9) to three sets of relic data are shown in Fig. 2. The flux profile for the Sausage relic is taken from van Weeren et al. (2010), for the NW relic in El Gordo from Lindner et al. (2014), and the Coma relic data from Trasatti et al. (in prep.; see Erler et al. 2015). We normalize each data peak to unity and use the published instrument resolutions to beam-convolve our profiles. When fitting the edge-on relics directly to their on-sky brightness profiles, we require parameter values roughly as $[\mu, \sigma]=\left[3.2,0.8 \alpha_{\text {tot }}\right]$, whereas fitting the volume emissivity profile requires much narrower distribution, roughly $[\mu, \sigma]=\left[0.1,0.3 \alpha_{\text {tot }}\right]$ (these values are for the three cases only, and should not be considered as general relic properties). The projection provides the requisite broadening in the 3D case, and hence the underlying lognormal profile is narrower. For the Sausage relic we note an excess emission "tail" in the downstream direction, which is inevitable because of our assumed circular geometry if the relic diameter (i.e., its LLS) is large (1.7 Mpc for Sausage). Therefore, for specific cases like the Sausage relic we fit the projected brightness for better accuracy, although our results do not change significantly when using a 3D emissivity model. For the Coma data, an edgeon relic cannot provide a good fit, so we use the 3D emissivity

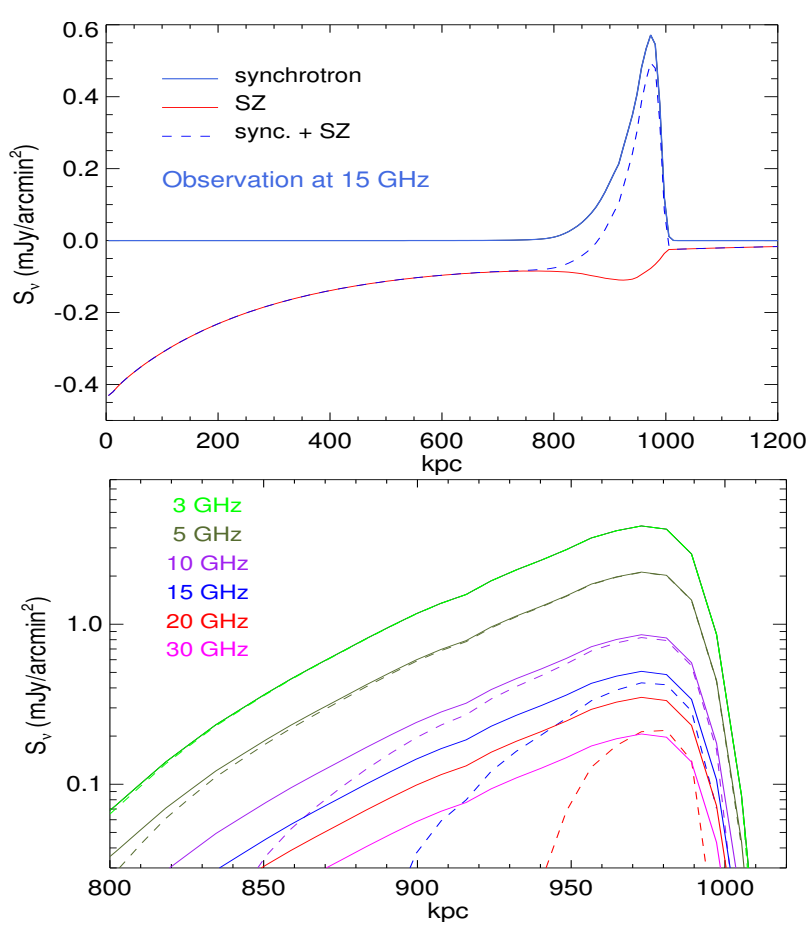

Fig. 3. Illustration of the SZ and synchrotron flux profiles across the location of a relic shock at $1 \mathrm{Mpc}$. The construction of the SZ and synchrotron fluxes are described in Sects. 2.1 and 2.3, respectively, and the example cluster is similar to the Sausage relic cluster. In the top panel we show the case at a single frequency $(15 \mathrm{GHz})$ for the full projected scale length of the cluster. The blue dashed line shows the modified radio flux. In the bottom panel the true and observed synchrotron fluxes are shown in solid and dashed lines in log scale. At $30 \mathrm{GHz}$ the signal is negative throughout because of the SZ decrement, although interferometric observation will be able to recover some positive signal (see Sect. 2.5). For relics with significant inclination angles the synchrotron profile is modified while keeping the SZ unchanged (Sect. 3.1.2).

model with a viewing angle of roughly $\sim 30^{\circ}$ (Fig. 2). Similarly, large viewing angle is needed for fitting the relic data in A2256, as we discuss in Sect. 3.2.5. We note that there is significant degeneracy between the lognormal model parameters with the viewing angle, so our fit results should not be considered unique solutions to these systems, but rather an approximation to match the observed radio data realistically.

A comparison for the relative amplitudes of the SZ and synchrotron fluxes is shown in the top panel of Fig. 3, for a fiducial shock model in a cluster of mass $6 \times 10^{14} M_{\odot}$ at $z=0.2$, shock Mach number $\mathcal{M}=3$, and shock radius $1 \mathrm{Mpc}$. The synchrotron profile is obtained through projection of a $3 \mathrm{D}$ edge-on relic model with lognormal emissivity profile, and matched to a total synchrotron power $P_{1.4}=4 \times 10^{24} \mathrm{~W} / \mathrm{Hz}$. In the bottom panel of Fig. 3 positive sections of the modified synchrotron+SZ flux profiles are shown with dashed lines, which we compare with the true synchrotron signal when computing contamination values. One simplifying assumption in our modeling is that the radio flux profile retains its shape at all frequencies, i.e., we ignore the spectral index variation across the relics and only use a global mean value, as this assumption does not change our results in any major qualitative way.

Figure 4 presents an example of how the synchrotron and SZ signals appear together after projection. The lognormal emissivity model follows the same $3 \mathrm{D}$ spherical shock geometry as discussed in Sect. 2.1 for computing the SZ signal. The crucial distinction from the $\mathrm{SZ}$ shock model is that synchrotron emission 


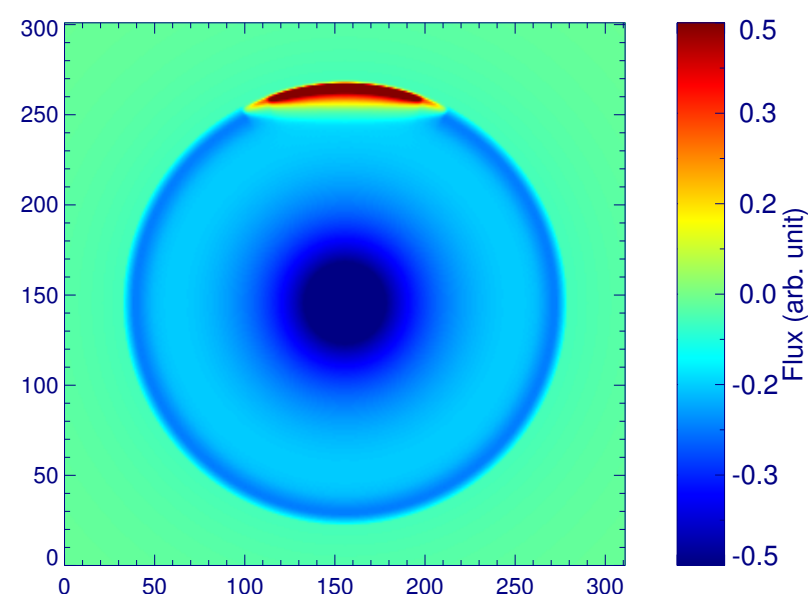

Fig. 4. Example of a $2 D$ image used for interferometric simulation The synchrotron and SZ emissivity models are placed on a spherical shock and projected, on top of a cluster-wide SZ signal that follows the GNFW pressure model. In this case the relic is viewed edge-on $\left(0^{\circ}\right.$ inclination angle). The full-spherical shock model for SZ is obvious, although for our purposes of computing flux contamination it is sufficient for the SZ signal boost to be localized in the same angular segment as the radio relic emission.

is localized within a circular patch whose diameter is determined by the measured relic linear scale (LLS). It is an observational fact that radio relics are localized emissions, even rare objects such as the Sausage relic do not trace a complete spherical shock front. One possibility is that this is due to an efficient electron acceleration in patches of magnetic fields that are perpendicular to the shock normal (e.g., Guo et al. 2014b,a). In fact, for relics that are viewed edge-on it is sufficient to have the SZ shock to be localized in the same region as marked by the synchrotron signal, since in projection the SZ signal boost gets its contribution mostly from a small region near the edge of the shock front. For relics viewed at large inclination angles the SZ signal modeling is generally more uncertain as the actual geometry of the shock front has more relevance.

\subsection{Side topic: $S Z$ contamination for radio halos}

Unlike radio relics, the issue of SZ flux contamination has already been considered in the context of radio halos (Enßlin 2002; Pfrommer \& Enßlin 2004; Brunetti et al. 2013). They have similar steep synchrotron spectrum as in relics, so at $\mathrm{GHz}$ frequencies their observed flux is likewise affected by the SZ effect. Here the standard picture of "negative bowl" of SZ effect applies: radio halos sit near the cluster centers where the SZ signal is strongest and both of these signals can have similar radial profiles (Planck Collaboration X 2013). The highest frequency measurement of a radio halo is at $\sim 5 \mathrm{GHz}$, for the nearby Coma cluster at $z=0.02$ (Thierbach et al. 2003). Brunetti et al. (2013) have argued that at $5 \mathrm{GHz}$ the effect of SZ decrement is negligible, accounting for less than $10 \%$ of the total synchrotron flux at that frequency and hence cannot be responsible for the observed steepening of the radio spectrum. Using the GNFW model (Eq. (5)) fit for Coma (Erler et al. 2015), we can confirm that the SZ contamination is indeed $\lesssim 10 \%$ at $5 \mathrm{GHz}$, so no current radio halo data has been seriously affected by this issue.

However, because of the redshift independence of SZ brightness, the flux contamination for radio halos will increase rapidly with redshift. We consider the famous El Gordo cluster
(ACT-CL J0102-4915) as an example, which at $z=0.87$ is currently the highest redshift radio halo known. Its highest frequency radio measurement is at $2.1 \mathrm{GHz}$ (Lindner et al. 2014), and using the GNFW pressure model appropriate for the mass and redshift for this cluster, we find a flux contamination value of only $4 \%$ within the radio halo radius $\left(r_{\mathrm{H}}\right.$ is similar to $r_{500}$, roughly $1.2 \mathrm{Mpc}$ ). If El Gordo radio halo is observed at $5 \mathrm{GHz}$ as in Coma, the contamination increases to $55 \%$, i.e., the observed synchrotron flux would be roughly half of the true flux (SZ flux roughly $-0.5 \mathrm{mJy}$ compared to extrapolated synchrotron flux $0.9 \mathrm{mJy}$ at $5 \mathrm{GHz}$, using a spectral index $\alpha=1.2$ ). We obtain similar high contamination values at $5 \mathrm{GHz}$ for other high- $z$ radio halos/mini-halos. For the Phoenix cluster at $z=0.6$ (van Weeren et al. 2014a), contamination is roughly $13 \%$ within the small mini-halo radius of $\sim 500 \mathrm{kpc}$. For the giant radio halo in PLCK G147.3-16.6 at $z=0.65\left(r_{\mathrm{H}} \sim 1 \mathrm{Mpc}\right.$; van Weeren et al. 2014b), the flux contamination at $5 \mathrm{GHz}$ is around $37 \%$. We do not elaborate further on this issue of radio halo flux contamination, but rather return to radio relics and their flux measurements.

\subsection{Interferometric and single-dish observations}

Radio data are obtained from either interferometric or singledish observation. The advantage of interferometers is their high angular resolution, which helps to remove point source contamination from the relic images. Therefore, also for single-dish imaging, it is desirable to have complimentary interferometric data for separating the compact emission. Interferometers, on the other hand, miss some large-angle flux on scales larger than $\sim \lambda / D_{\min }$, where $D_{\min }$ is the smallest baseline length. For $\mathrm{cm}$ wavelength observations $(1-30 \mathrm{GHz})$ this is particularly a problem, as there are not many interferometers with short enough baselines that can image scales of several arcminutes, as is often exhibited by radio relics. Indeed, the two interferometers that have been used for $\mathrm{cm}$ wavelength relic imaging (AMI and CARMA) were specifically designed to image the cluster-wide SZ signal at these wavelengths. Loss of signal as a result of a lack of sufficiently short spacings can be a problem for all known relics at $\mathrm{cm}$ wavelengths, save for compact high- $z$ sources such as the El Gordo relic (whose length is $\lesssim 1$ arcmin). Single-dish measurements have mostly been performed by the Effelsberg $100 \mathrm{~m}$ radio telescope, using its $3-10 \mathrm{GHz}$ bands (Trasatti et al. 2015; Stroe et al. 2016).

The basic difference between single-dish and interferometric imaging is that the former measures total power (depending upon the scan length), whereas the latter is sensitive to the signal autocorrelation in the plane of the sky, i.e., measures the change in brightness. It might be assumed that since the SZ signal is generally large scale compared to the localized synchrotron emission, the effect of interferometric imaging would be to remove the large-scale SZ flux while keeping the synchrotron part intact, thereby recovering the true synchrotron signal (assuming the relic to be sufficiently compact). While this would be the case if the radio relic was situated somewhere in the negative "bowl" of the cluster-wide SZ signal, the shock also creates a sharp SZ flux variation on the same scale as radio relics (top panel of Fig. 3), thus single-frequency interferometric imaging are not able to disentangle the synchrotron signal from the SZ component. How much of the radio relic flux is actually recovered depends upon the details of the image deconvolution process, the $u v$-coverage in relation to the scale of the emission, and the signal-to-noise ratio of the measurement. 


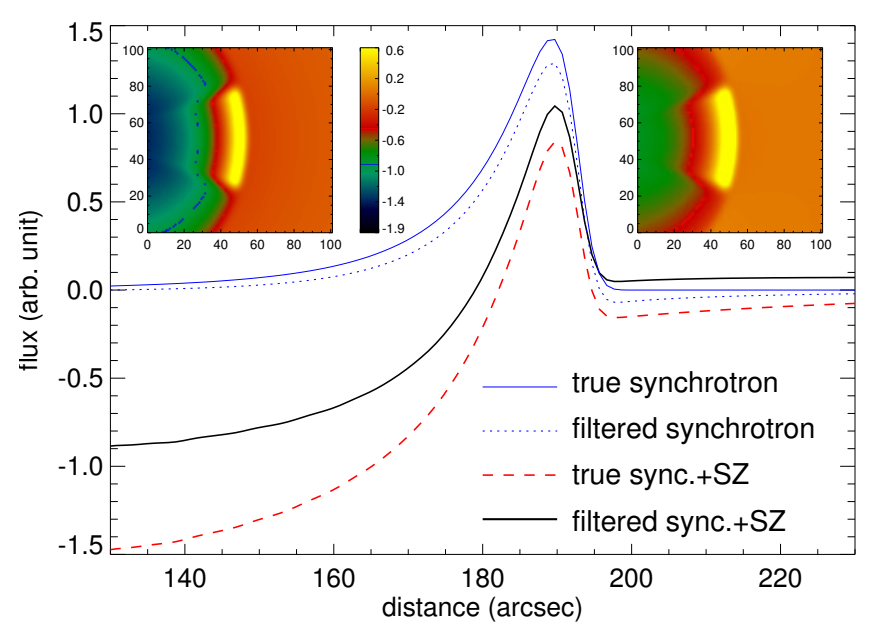

Fig. 5. High-pass filtering of a $2 D$ radio relic model in the presence of SZ decrement, done as a simplified exercise for interferometric observation. The inset panels show the input image before (left) and after (right) filtering, using the same color scale. The multiple curves in the main panel plot represent the profiles for synchrotron-only model (blue solid line), filtered synchrotron model (blue dotted line), synchrotron+SZ model (red dashed line), and filtered synchrotron+SZ model (black solid line). We see that the high-pass filter effectively removes the large-scale SZ signal from the image, while keeping the measured peak flux close to the input (unfiltered) value of the synchrotron+SZ model, and not the "synchrotron only" model.

\subsubsection{High-pass filtering of the total signal}

We first consider a simple exercise of high-pass filtering of a noiseless radio relic image, in the presence of negative SZ signal, to get an intuitive understanding of imaging with interferometers. The input image is a simplified radio synchrotron relic plus SZ model, shown in the left inset of Fig. 5. Onto this we apply a high-pass filter (Butterworth filter of first order), whose cutoff frequency is designed to keep the original relic signal mostly unchanged. The image after filtering is shown in the right inset with the same color scale. The radial profiles before and after the high-pass filtering is shown in the main panel of Fig. 5. Here the blue solid line is the synchrotron signal without any SZ effect, and the red-dashed line is the synchrotron+SZ signal that an ideal total-power imager finds. The filtered profile is shown by the solid black line that is the result of an interferometric observation. A filtered synchrotron-only profile is shown with a blue dotted line, which is lower than the input synchrotron model.

Three things are to be noted from this exercise of high-pass filtering. First, the large-scale SZ signal is mostly removed from the filtered image, and the peak of the measured signal (black solid line in Fig. 5) corresponds closely to the modified synchrotron signal (red dashed line), meaning SZ-shock induced flux modifications are there also after filtering. Second, the filtering would also affect the original synchrotron signal to some extent (blue dotted line), meaning there would also be some flux loss without SZ contamination. Third, and more subtly, the filtering process compensates for some of the flux loss by removing the large-scale negative SZ component, as expected from a highpass filter (more positive flux under the black solid line than the red dashed line). As we show in the next subsection, the situation is somewhat altered when we consider a more realistic simulation of an interferometric observation.

\subsubsection{Simulated interferometric observation}

In terms of radio interferometry, a high-pass filtering corresponds, to a first approximation, to an inverted (or "dirty") image in presence of infinite signal-to-noise and full coverage of the unfiltered part of the $u v$-plane. In the case of real observations, one typically uses the CLEAN algorithm to do the imaging, "filling in" the missing information in the $u v$-space by interpolating from the existing data, thus producing an image with minimal artifacts (see Taylor et al. 1999 for details). In a radio interferometric observation, flux can also be recovered on scales much larger than expected from a simple consideration of the shortest baseline. For example, if one dimension of the source is much larger than the other (as in most radio relics), a mosaic of several pointed observations can recover the full flux of the source, given that the smaller dimension is within range of the interferometer array. However, such signal reconstruction is satisfactory only in the limit of very high signal-to-noise, and an almost complete $u v$-coverage (full rotation synthesis).

The reconstruction of the modified relic signal is more complicated, as there will be both positive and negative signal components. For moderate signal-to-noise cases (cm wavelength relic observations typically have $S / N \sim 5$ for the peak flux), one would generally apply the CLEAN algorithm by selecting regions ("clean boxes") around sources. In case of radio relics it can be natural to select regions which only show a positive residual flux in a noisy field. Thus the outcome of the image deconvolution will vary depending on the CLEAN regions applied, as well as the relic shape and size and the signal-to-noise of the measurement.

To make realistic simulations for interferometric imaging, we employ the $\mathrm{CASA}^{1}$ software package, in particular its SIMOBSERVE task, to create a measurement set from the model image (similar to Fig. 4), which is then deconvolved using CLEAN with the Briggs weighting (robust=1) scheme. The clean mask is defined from the signal-to-noise ratio of the residual image, cleaning only in regions where this ratio is greater than five. These parameters are chosen to minimize the flux loss from the diffuse relic signal, and cleaning is performed interactively. We simulate a VLA $X$-band $(10 \mathrm{GHz})$ observation in D-configuration, and considering the limited spacial fidelity of VLA at these frequencies (largest recoverable scale $\sim 2$ arcmin), we make a compact relic model similar to the NW relic in the El Gordo cluster, such that the angular extension of the relic is roughly 1.5 arcmin and one would not need mosaicking. The thermal noise level from the simulator is chosen to have a peak $S / N \sim 10$ in the image. We simulate both a "synchrotron-only" model and a "synchrotron plus SZ" model, with the same noise realization, to compare the fluxes after CLEANing. Primary beam correction is taken care of by the SIMOBSERVE task.

The simulated observations are shown in Fig. 6. The top panels show the CLEANed images of radio synchrotron signal in presence of SZ flux decrement (left), and only the radio synchrotron signal (right). The flux loss in the presence of SZ is evident, both from a reduced peak value and a shrunken width. The curves in the lower panel show the observed flux profiles in comparison to the input models. The black dashed line is the input model of synchrotron plus SZ, which is observed by an ideal total-power instrument. The actual interferometric observation is the black solid line, missing the SZ negative parts completely, and yielding more positive flux than the model. This is to be compared with the input synchrotron-only model (red dashed

1 http://casa.nrao.edu/ 

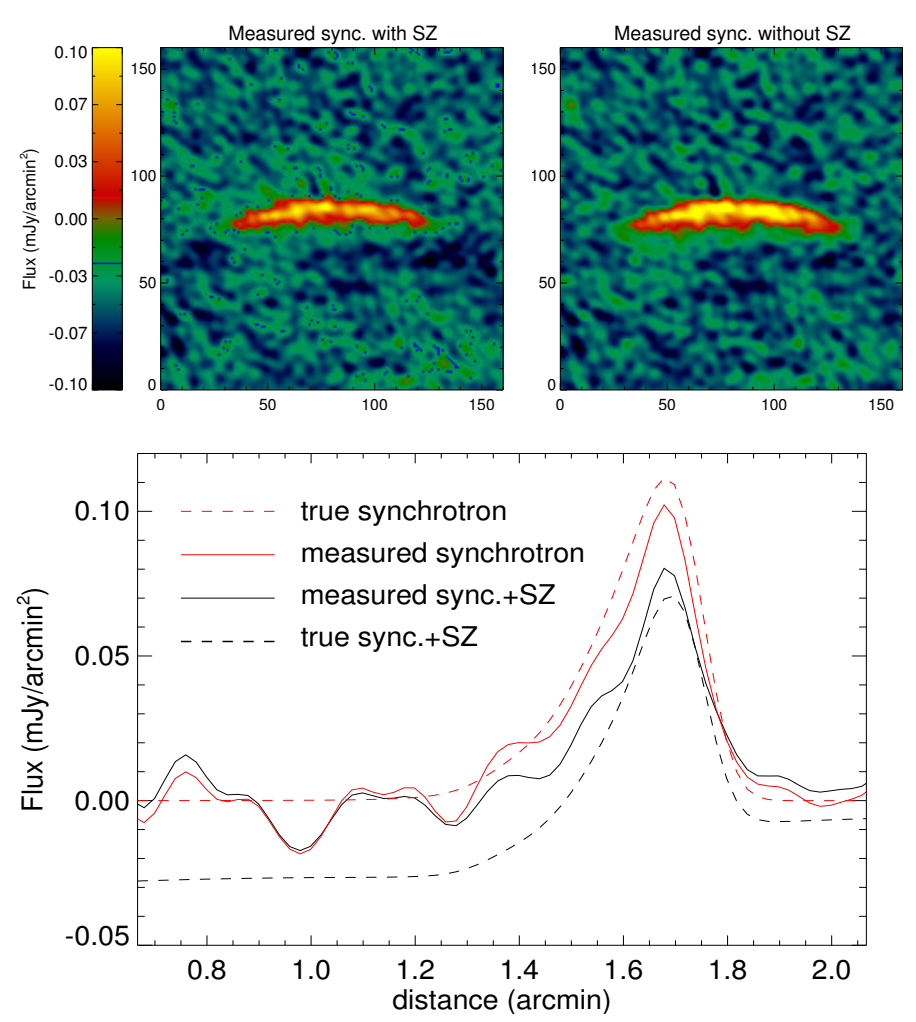

Fig. 6. Simulated interferometric observation at $10 \mathrm{GHz}$ for an El Gordo-type compact relic with the VLA $X$-band. Simulations are performed by the CASA software and CLEANed interactively. Top panel left: actual measurement of the radio relic in the presence of SZ flux contamination. Top panel right: hypothetical measurement of only the synchrotron emission without any SZ. The image axes are in arcseconds, both cases use the same noise realization, and are shown in the same color scales. Bottom panel: flux profiles before and after processing through the simulation setup. The red dashed line shows the input synchrotron-only model, and the black dashed line indicates the synchrotron+SZ input model. Red solid line shows the effect of interferometer on a synchrotron-only flux measurement, which also registers some flux loss due to lack of short baselines. The black solid line refers to the actual observed flux with interferometers when $\mathrm{SZ}$ is also taken into account.

line), which also suffers from flux loss because of a lack of sufficient short baselines. To illustrate by numbers: the total flux in our input synchrotron model at $10 \mathrm{GHz}$ is $0.22 \mathrm{mJy}$, and the total positive flux in presence of SZ decrement is $0.11 \mathrm{mJy}$. So the flux contamination is $50 \%$ for an ideal observation. We define the contamination fraction as the ratio between the flux lost due to the SZ effect and the true (uncontaminated) synchrotron flux. Thus the contamination (in percentage) is $C=100 \times$ (true flux measured flux)/true flux, which makes it roughly equivalent to the flux ratio: $C \approx 100 \times(\mathrm{SZ}$ flux/synchrotron flux $)$. In the case of simulated interferometric imaging with CLEANing, the recovered synchrotron-only flux is $0.18 \mathrm{mJy}$, and the synchrotron flux in presence of SZ contamination is $0.13 \mathrm{mJy}$. So the interferometric observation produces a flux contamination of roughly $40 \%$, somewhat less than our idealized value. This flux change is due to both the presence of SZ signal (majority contribution) and the loss of signal resulting from missing short spacings for the faint and extended source.

To summarize, interferometric imaging of radio relics at $\mathrm{cm}$ wavelengths will register flux loss similar to a single-dish measurement because of the presence of a relic-scale negative SZ feature from the shock, and also, by a smaller amount, because of the insufficient $u v$-coverage when imaging a large, diffuse relic emission. From the particular case analyzed with our mock interferometric observation, we found that the combined effect of these two modifications can be roughly equal to the diminished flux measured by an "ideal" total-power instrument, that registers both the positive and negative fluxes accurately. We can assume that flux losses due to inadequate short baselines are an almost inevitable feature for high-frequency radio imaging, unless compact, high- $z$ relics are targeted or special care is taken to combine interferometric results with single-dish data ("zero-spacing" addition). The actual amount of flux that is recovered by any specific interferometer is a complicated function of signal-to-noise, the source size and shape, amplitude of the ambient SZ signal, etc., and is difficult to predict. It will also not scale as a fixed fraction of the ideal contamination number owing to the changing shape of the flux profile with frequency. Therefore, in our presentation of the contamination results in this paper, we quote contamination values simply by comparing the true positive synchrotron-only fluxes with the measured positive radio fluxes after adding the $\mathrm{SZ}$ decrement.

\section{Results}

In the first Sect. 3.1 we present some mean trends for the flux contamination: namely how it varies with redshift, cluster mass, shock Mach number, and also the relic viewing angle. In the following Sect. 3.2 we provide results for some well known relics in individual clusters. Results from the latter section are summarized in Table 1.

\subsection{Modeling results for the mean contamination}

First we use the GNFW cluster model (Eq. (5)) and an empirical scaling relation between a cluster's mass and its radio relic power, to predict the general trend of the SZ flux contamination with cluster mass and redshift. We also describe, using simplified models, how the SZ-to-synchrotron flux ratio is expected to vary with shock Mach number and use the rotation of the relic model described in Sect. 2.3 to qualitatively outline the impact of relic viewing angles.

\subsubsection{SZ contamination as function of redshift}

As the SZ surface brightness is practically redshift independent, the flux contamination increases rapidly with redshift. What is somewhat unexpected is that for higher mass systems we get less contamination. This is because of the strong mass dependence on the radio relic luminosities that is found empirically $\left(P_{1.4} \propto M_{500}^{2.8}\right.$; de Gasperin et al. 2014), which is steeper than the mass dependence of the cluster SZ signal $\left(Y_{\mathrm{SZ}} \propto M_{500}^{5 / 3}\right)$. Assuming the shock Mach number and magnetic field intensity to be independent of cluster mass, this means that for lower mass systems the synchrotron power falls off more quickly than the SZ signal, causing more significant contamination. We do not attempt to explain this strong mass dependence of radio relic power; see de Gasperin et al. (2014) for a discussion on this issue. However, we note that similar strong mass dependence is also found for radio halos (e.g., Sommer \& Basu 2014), and there too the SZ flux contamination should decrease with increasing mass. 
K. Basu et al.: SZ contamination in radio relics

Table 1. SZ flux contamination predictions, in percentage, for some well-known radio relics at different frequencies.

\begin{tabular}{rcccccc}
\hline \hline & $3 \mathrm{GHz}$ & $5 \mathrm{GHz}$ & $10 \mathrm{GHz}$ & $15 \mathrm{GHz}$ & $20 \mathrm{GHz}$ & $30 \mathrm{GHz}$ \\
\hline Sausage relic $(\mathcal{M}=2.5)$ & $<1 \%$ & $<1 \%$ & $4 \%$ & $11 \%$ & $24 \%$ & $58 \%$ \\
$(\mathcal{M}=3.5)$ & $<1 \%$ & $<1 \%$ & $3 \%$ & $10 \%$ & $21 \%$ & $49 \%$ \\
$(\mathcal{M}=4.5)$ & $<1 \%$ & $<1 \%$ & $4 \%$ & $12 \%$ & $24 \%$ & $52 \%$ \\
& & & & & & \\
Toothbrush relic $(\mathcal{M}=3.5)$ & $<1 \%$ & $<1 \%$ & $3 \%$ & $9 \%$ & $18 \%$ & $43 \%$ \\
$(\mathcal{M}=4.5)$ & $<1 \%$ & $<1 \%$ & $3 \%$ & $10 \%$ & $20 \%$ & $46 \%$ \\
El Gordo relic $(\mathcal{M}=2.5)$ & $<1 \%$ & $3 \%$ & $23 \%$ & $53 \%$ & $81 \%$ & $>100 \%$ \\
A2256 relic $(\mathcal{M}=2.0)$ & $1 \%$ & $3 \%$ & $28 \%$ & $66 \%$ & $96 \%$ & $>100 \%$ \\
Coma relic $(\mathcal{M}=2.2)$ & $<1 \%$ & $<1 \%$ & $6 \%$ & $21 \%$ & $41 \%$ & $82 \%$ \\
\hline
\end{tabular}

Notes. We define the contamination value as $C(\%) \equiv 100 \times$ (true flux - measured flux)/true flux. The flux correction factor, $F$, which one should multiply to get the true synchrotron flux values, is therefore $F=100 /(100-C)$. From this definition, $C$ is equal to the flux ratio between SZ and

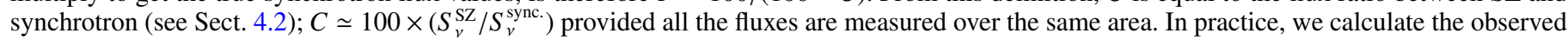
radio flux by integrating over the area where relic emission is positive, approximating an ideal total-power measurement. A contamination value of more than $100 \%$ means that the synchrotron flux is completely overshadowed by the negative SZ signal.

We fix the normalization of the empirical scaling result from de Gasperin et al. (2014) as follows:

$P_{1.4} \approx 1.3 \times 10^{24}\left(\frac{M_{500}}{4 \times 10^{14} M_{\odot}}\right)^{2.8}\left(\mathrm{~W} \mathrm{~Hz}^{-1}\right)$.

Apart from the considerable scatter in this scaling relation, this also neglects all possible evolution of cluster properties relevant for the radio synchrotron emission. For SZ we used the selfsimilar redshift dependence $E(z)^{8 / 3}$ (Eq. (5)) for scaling. Thus the above scaling law is clearly an approximation, but even for fixed luminosities for both synchrotron and the SZ signal, their observed flux ratio scales as $(1+z)^{4}$ (the ratio $\left.D_{\mathrm{L}}^{2} / D_{\mathrm{A}}^{2}\right)$ on top of which there is a strong $K$-correction term due to the steeply falling synchrotron spectrum. Thus, a strong $z$-increase in the flux contamination is also inevitable with a more precise synchrotron power scaling relation.

In Fig. 7 we show examples of the SZ flux contamination increase with redshift for two sets of cluster masses: $M_{500}=$ $4 \times 10^{14} M_{\odot}$ and $1 \times 10^{15} M_{\odot}$, using a relic model with $\mathcal{M}=2.5$ shock at $r_{\text {shock }}=1 \mathrm{Mpc}$. It is seen that for $15 \mathrm{GHz}$ observation the measured relic fluxes can be less than half of its true value at $z=0.5$ and above. This is important for several currently known intermediate- to high- $z$ relics, one such example is discussed in Sect. 3.2.3 for the El Gordo cluster.

\subsubsection{Effect of the viewing angle}

For most of our work we assume that radio relics are viewed edge-on, i.e., the cluster merger and shock propagation is happening in the plane of the sky. While this is a reasonable assumption for double radio relics, for many other single relic observations the viewing angle remains an open and important issue. Prominent examples are the relics in the Coma and A2256 clusters, which are discussed later. We briefly present a qualitative description of the effect of changing viewing angles in the framework of our lognormal emissivity model on top of a spherical shock.

Clearly, as the relic is viewed more and more obliquely (toward or away from the observer, the cases are symmetric), its peak flux amplitude drops while keeping the total flux conserved. If we assume a more extended spherical shock front for the

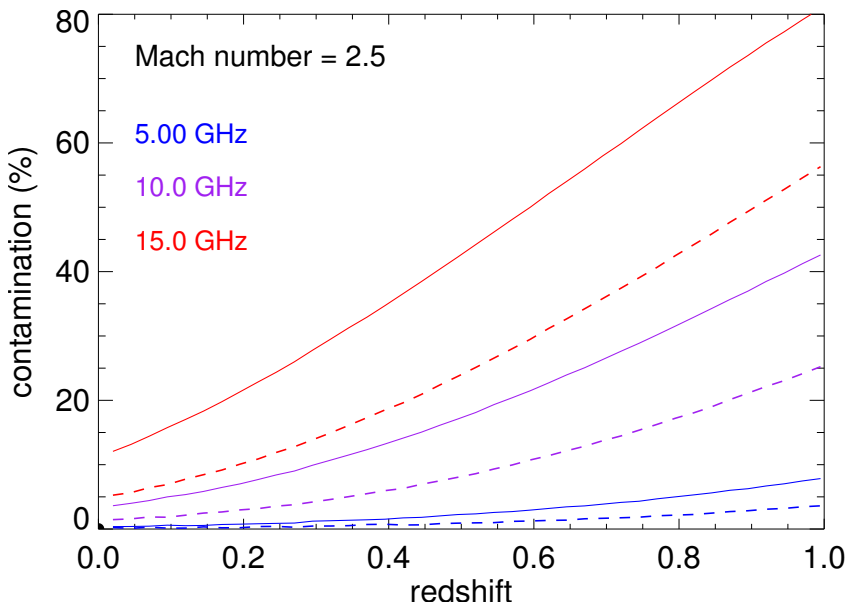

Fig. 7. SZ contamination in measured radio fluxes with increasing redshifts for two different cluster masses. The solid lines are for a cluster with mass $M_{500}=4 \times 10^{14} M_{\odot}$, and the dashed lines are for a higher mass, $M_{500}=1 \times 10^{15} M_{\odot}$. Three different colors correspond to three observing frequencies, and we use a $\mathcal{M}=2.5$ shock with relic LLS $=0.5 \mathrm{Mpc}$, at $1 \mathrm{Mpc}$ cluster-centric distance. We use an empirical relic power-to-cluster mass scaling, $P_{v} \propto M^{2.8}$, from de Gasperin et al. (2014), which is stronger than the cluster SZ signal scaling. Hence the contamination decreases with increasing cluster mass.

SZ signal, this also means that the peak of the relic synchrotron flux moves away from the SZ signal jump, which is unaffected by rotation. This scenario is depicted in Fig. 8, using the SZ and synchrotron signal modeling described earlier, but with an additional rotation perpendicular to the plane of the sky prior to projection. Detailed shapes of these profiles depend on numerous factors, such as the relic opening angle and curvature, and the flux profile along the length of the relic, etc. What concerns us is the approximate shape with respect to the shock-front location. We can see that for even a mild rotational angle of $\sim 20^{\circ}$ the peak flux drops by roughly a factor two. Additionally for the flux contamination issue, the bulk of the synchrotron emission shifts away from the SZ "jump" at the shock front, where the SZ signal is more uniformly negative. For a single-dish instrument we get more flux contamination as the SZ signal is generally stronger 


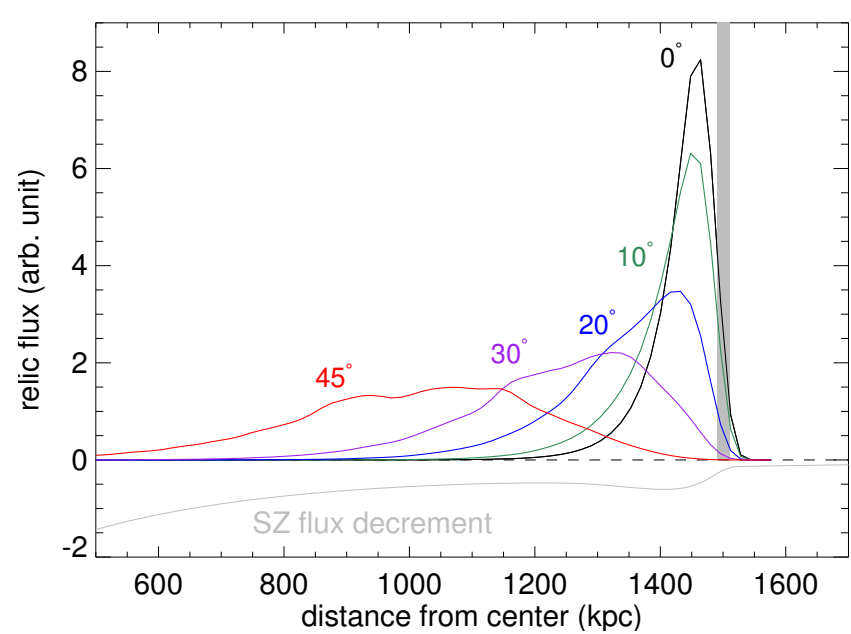

Fig. 8. Radio relic with a lognormal emissivity model from different viewing angles. For this illustration we use a relic with dimension $500 \mathrm{kpc}$ at a distance of $1.5 \mathrm{Mpc}$ from a cluster center, situated at $z=0.2$, and simulate an observation with resolution $10^{\prime \prime}$. The gray vertical band shows the location of the shock front and a negative SZ flux profile is included for comparison.

near the cluster center, but an interferometer generally filters out this uniform SZ signal and recovers close to the true synchrotron flux. We present total-power flux contamination estimates for the Coma and A2256 relics in Sect. 3.2.

\subsubsection{Effect of changing Mach numbers}

Any change in the Mach number distribution at shocks affects the relic synchrotron emission in a complicated way. In the simple scenario of single injection by a merger shock, we can assume that radio synchrotron power scales as $\mathcal{M}^{3}$, as the particle energy injection is proportional to the kinetic power dissipated by the shock: $E_{\text {kin }}=\mathcal{M}^{3} n_{\mathrm{u}} c_{s}^{2} A / 2$ (e.g., Vazza \& Brüggen 2014; where $n_{\mathrm{u}}$ is the upstream density, $c_{\mathrm{s}}$ is the sound speed, and $A$ is the shock surface area). In such a simplistic model the synchrotron-to-SZ flux ratio then scales linearly with $\mathcal{M}$, since the SZ flux change is roughly proportional to $\mathcal{M}^{2}$ at the shock front (Eq. (6)). Thus we can expect a decrease in flux contamination in radio relics with higher Mach numbers.

A more sophisticated modeling can be obtained through the formalism by Hoeft \& Brüggen (2007) that attempts to describe the stationary power law emission, starting from the electrons in the Maxwellian tail that are energetic enough to enter diffusive shock acceleration. This model introduces an efficiency function that takes into account the particle spectra as a function of $\mathcal{M}$ to compute the total injected energy into the electron population. A useful polynomial expression for this is given by the function $\eta(\mathcal{M})$ in Kang et al. $(2007)^{2}$. This efficiency function has been derived for protons, and within the uncertainties related to the modeling of shocks it is also appropriate to use it to derive the electron acceleration efficiency, by rescaling for the electron-toproton ratio, $\xi_{\mathrm{e} / \mathrm{p}}=0.05$ (Kang \& Ryu 2013). The characteristic feature of $\eta(\mathcal{M})$ is a very steep drop below $\mathcal{M} \lesssim 3$, and a roughly constant value above $\mathcal{M} \gtrsim 6$. With such a dependence we get

\footnotetext{
2 Compared to the original work by Hoeft \& Brüggen (2007), the $\eta(\mathcal{M})$ function by Kang et al. (2007) also takes into account the gas compression at the shock and must be applied to upstream quantities. On the other hand, the efficiency given in Hoeft \& Brüggen (2007) is meant to be referred to downstream quantities. We follow the convention by Kang et al. (2007) and, therefore, make use of upstream gas quantities.
}
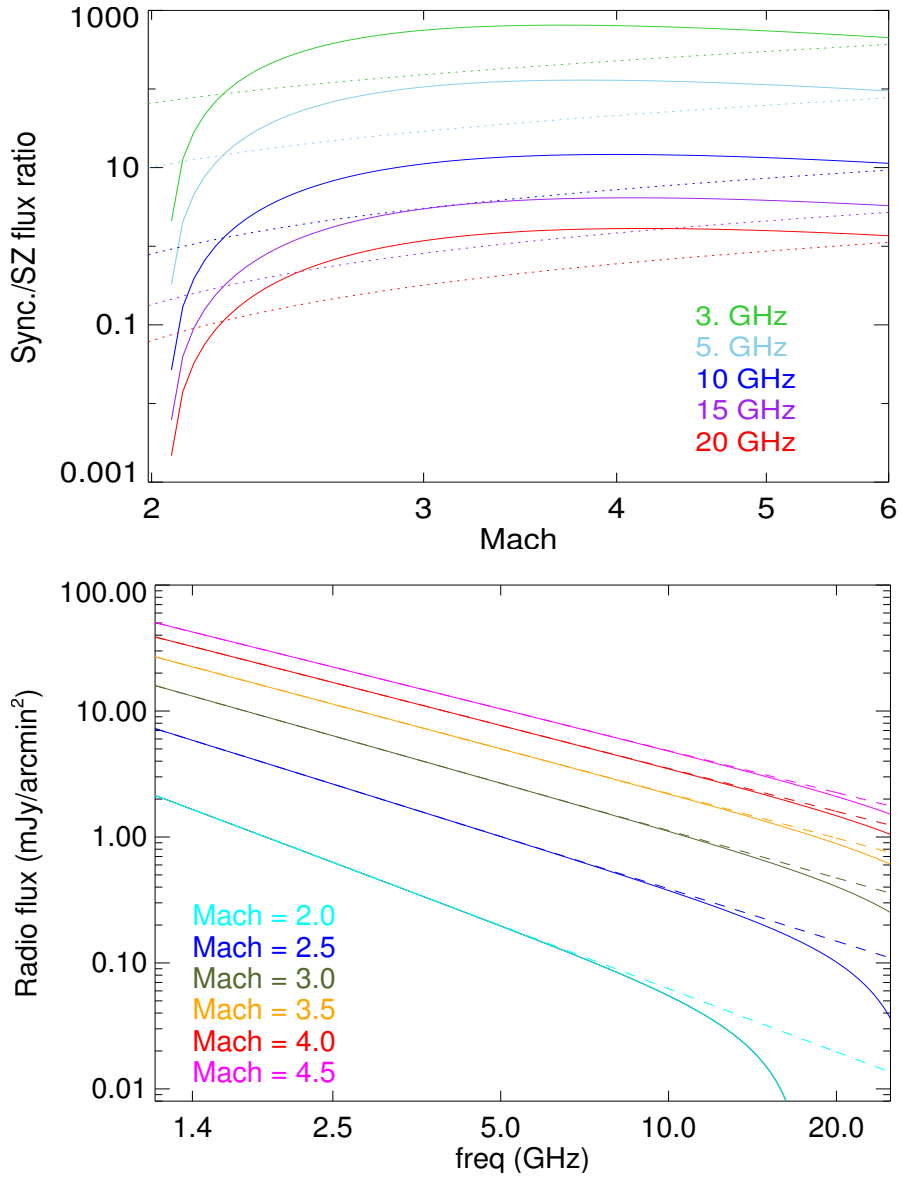

Fig. 9. Effect of a change in Mach number onto the measured radio spectrum. Top panel: synchrotron-to-SZ flux ratio as a function of the shock Mach number, seen at different observing frequencies. In the DSAbased model this ratio varies rapidly only below $\mathcal{M} \lesssim 2.5$, above which it asymptotes to a constant ratio that starts to decrease at high $\mathcal{M}$-values. For comparison, results from a simple $\mathcal{M}^{3}$ dependence of the radio synchrotron power is shown with dotted lines, where the flux ratio increases linearly (see Sect. 4.2). Bottom panel: the observed radio spectra for different Mach number values, using the linear Mach dependence of the synchrotron-to-SZ flux ratio. The dashed lines are uncontaminated synchrotron spectra that follow a single power law throughout.

a synchrotron-to-SZ flux ratio as shown in Fig. 9 top panel. We also show the simplistic picture of $P_{\text {sync. }} \propto \mathcal{M}^{3}$ with dotted lines, where the flux ratio is linear. In the bottom panel of Fig. 9 we show the resulting spectrum for radio observation at $\mathrm{cm}$ wavelengths. The increase in the low-frequency synchrotron flux amplitudes with increasing Mach numbers from the linear model is evident.

The discussion above ignores several complications of the particle acceleration by merger shocks, which are yet to be solved (e.g., Vazza et al. 2015 and discussion therein). The presence of reaccelerated electrons can contribute to the synchrotron emission together with the freshly accelerated electrons, although this contribution is important only for $\mathcal{M} \leq 3$ shocks (Pinzke et al. 2013). Amplification of the magnetic fields by cosmic rays-induced turbulence is expected to be important only for $\mathcal{M} \geq 10$ (Brüggen 2013) and therefore can be neglected. In Sect. 4.2 we discuss some approximations to the DSA-based model to match its predictions to real radio relic data, where we neglect these secondary effects. 


\subsection{Modeling results matching actual relic data}

In this second part of our results we present detailed contamination modeling for some well-known radio relics, including the Sausage relic in CIZA J2242.8+5301 (Sect. 3.2.1) and the NW relic in the El Gordo cluster (Sect. 3.2.3). Whenever possible we use actual measured values for the Mach numbers and set the normalization of the GNFW pressure model to match the global SZ signals against measured values.

\subsubsection{The Sausage relic in CIZA J2248.8+5301}

We first present results for the flux contamination in the Sausage relic, as its $16 \mathrm{GHz}$ flux measurements (Stroe et al. 2014) were a main motivation for this work. The fit to the Sausage brightness profile with our lognormal model was discussed in Sect. 2.3, where we mentioned that, because of projection, the 1.7 Mpc length of the Sausage relic makes it difficult to obtain the sharp drop in radio synchrotron brightness seen in the post-shock end. To avoid this difficulty, we simply model the projected brightness profile (at $610 \mathrm{MHz}$; van Weeren et al. 2010) directly with a lognormal model, which fit the flux drop in the post-shock region very well, even though it is less physically motivated. Another difficulty is the choice of Mach number for our modeling. From the synchrotron spectral index van Weeren et al. (2010) derived $\mathcal{M}=4.6$, whereas $\mathrm{X}$-ray temperature measurements from the Suzaku satellite yielded distinctly lower values in the range 2.7-3.2 (Akamatsu \& Kawahara 2013; Ogrean et al. 2013; Akamatsu et al. 2015). We therefore present our results for three different Mach number possibilities $(\mathcal{M}=2.5,3.5,4.5)$. The total spectral index of the relic is derived from the chosen Mach numbers (Eq. (8)) instead of using some fixed value ${ }^{3}$. The other ingredients for computing the flux contamination are the relic length (LLS $=1.7 \mathrm{Mpc}$ ), distance from the cluster center $\left(r_{\text {shock }}=1.5 \mathrm{Mpc}\right)$, and the cluster mass.

For the Sausage relic cluster and other cluster results presented afterward, we make an additional correction for the cluster mass based on the Planck all-sky measurements. The cluster masses are generally first estimated from their X-ray luminosities, but as all these relic clusters are merging systems, a large deviation from the mean scaling relations can be expected. The published all-sky Planck data (Planck Collaboration XVI 2016) presents the possibility to calibrate the cluster SZ signal from $Y_{500}$ measurements directly. CIZA J2248.8+5301 is not in the published Planck catalog because of its proximity to the Galactic plane, and some other known relic clusters are absent either because they are low mass systems or too compact. To keep uniformity over all the cluster mass calibration results, we extract $Y_{500}$ by fitting a GNFW model to an $y$-map extracted from the Planck all-sky data (see Erler et al. 2015 for details). For CIZA $\mathrm{J} 2248.8+5301$ we obtain $Y_{500}=(3.6 \pm 0.1) \times 10^{-3} \mathrm{arcmin}^{2}$, which is about a factor 2 more than the GNFW model prediction based on the $L_{\mathrm{X}}$-derived mass $\left(M_{500}^{\mathrm{X} \text {-ray }}=5.5 \times 10^{14} M_{\odot}\right)$. The upwardcorrected mass is also very similar to the recent measurement for weak-lensing mass by Jee et al. (2016). Therefore, we scale the ambient pre-shock pressure north of the relic for a mass value in the GNFW model with $M_{500}=8.5 \times 10^{14} M_{\odot}$, with the assumption that the boost in $Y_{500}$ owing to the relic shock is small compared to the global SZ signal of the cluster.

\footnotetext{
3 Low-frequency data for the Sausage relic (and also the Toothbrush relic discussed in Sect. 3.2.2) are poorly fit by a single power law; see, for example, Stroe et al. (2016) for a discussion.
}
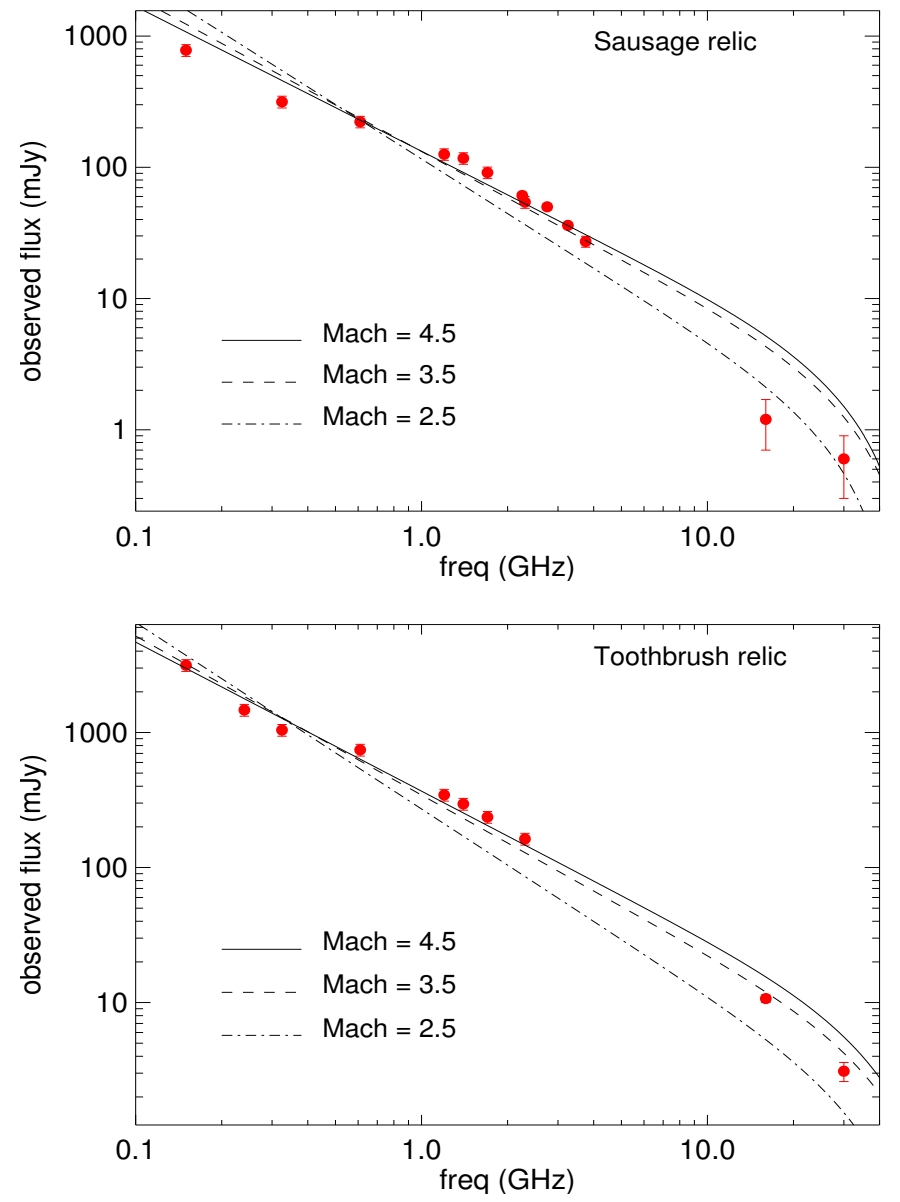

Fig. 10. Interferometric flux measurements of the Sausage relic in CIZA J2248.8+5301 (top panel) and the Toothbrush relic in 1RXS J0603.3+4214 (bottom panel) against our spectral predictions for three different shock Mach numbers. The data are taken from Stroe et al. (2016). The predicted spectra are normalized to have minimum $\chi^{2}$ computed from the low-frequency $(\lesssim 2 \mathrm{GHz}$ ) part. These model spectra correspond to an "ideal" measurement, and the results from actual interferometric imaging can differ (see text).

The comparison between the Sausage relic data and our spectral computations is shown in the upper panel of Fig. 10. The data points are interferometric measurements with comparable scales (Stroe et al. 2016). We compute the synchrotron fluxes based on the $610 \mathrm{MHz}$ data, but apply an additional scaling such that the low-frequency part of the spectrum provides the best possible fit to all the low-frequency data $(\lesssim 2 \mathrm{GHz})$. This way, our spectral prediction are not sensitive to any particular "pivot" flux value. For $\mathcal{M}=3.5$ we compute a contamination of less than $1 \%$ at $5 \mathrm{GHz}$, and roughly $14 \%$ at $16 \mathrm{GHz}$. This is within the $1 \sigma$ statistical uncertainty in the AMI measurement quoted by Stroe et al. (2014). Above this frequency the flux contamination increases rapidly; for the same Mach number we have roughly $25 \%$ flux contamination at $20 \mathrm{GHz}$, and $50 \%$ contamination at $30 \mathrm{GHz}$. In other words, the measured radio flux is roughly half of the true synchrotron value at $30 \mathrm{GHz}$. The AMI $16 \mathrm{GHz}$ measurement can be considered too low for our synchrotron+SZ spectral model, particularly as the single power law with $\mathcal{M}=2.5$ provides a poor fit to the low-frequency part. This might be indicative on an intrinsic spectral steepening, however, any such amplitude can only be robustly constrained after taking the SZ effect together with the relevant geometrical and observational parameters into account. 
At this level, our spectral modeling is not an accurate prediction for the specific interferometric measurements, in particular, the critical high-frequency results from AMI and CARMA. This requires a detailed knowledge of the interferometric setup and imaging procedures. The flux loss numbers shown here should be taken only as a guideline rather than exact predictions for the real-life interferometric observation. As we showed in Sect. 2.5, interferometers definitely compensate for some of the lost flux caused by the SZ decrement, but also miss flux on large scales, particularly for nearby relics like Sausage. The ideal flux contamination numbers shown here are not a lower limit either, as the flux loss due to a lack of short baselines can go beyond the change caused by SZ alone. In Sect. 2.5 we discussed the change of flux only in the transverse direction (for Sausage relic N-S direction), but the interferometric flux loss due to inadequate $u v$ sampling affects the longitudinal (E-W) direction more, and the measured flux reflects the combined effect. Following the discussion in Stroe et al. (2016), we do not use the single-dish "total power" spectra from their work as a result of the possibility of point source contamination and wrong baselevel corrections. On the physical side, prediction for the SZ decrement can vary along the relic length depending on inhomogeneous ICM distribution, Mach number variation, etc.

From the predicted contamination percentage values listed in Table 1, one can note an interesting trend. For various Mach number values assumed, the flux contamination (i.e., the ratio of synchrotron to SZ flux) is roughly constant at any given frequency. In Sect. 3.1.3 we saw that in a DSA-based modeling the flux ratio reaches a constant value above $\mathcal{M} \gtrsim 3$, but in the calculation for Sausage relic we have not assumed any specific model to predict the synchrotron flux. Instead, this behavior comes from computing the radio spectral index from the shock Mach number, which for our Mach-range of interest $(\mathcal{M} \sim 2-4)$ produces roughly $\alpha_{\text {tot }} \propto \mathcal{M}^{1 / 2}$. Thus when extrapolating synchrotron fluxes from a fixed measured value at low frequency, the log of synchrotron flux at a fixed higher frequency roughly scales as $\log \left(S_{\text {sync. }}\right) \sim \mathcal{M}^{1 / 2}$. The SZ flux boost, on the other hand, scales as $\mathcal{M}^{2}$, i.e., $\log \left(S_{\mathrm{SZ}}\right) \sim 2 \log (\mathcal{M})$. In the narrow Mach-range of interest these two functions produce roughly the same rate of change. Therefore, measuring the SZ flux contamination at one single frequency may not be a sensitive test for determining the shock Mach number. However, if the synchrotron spectral index can be robustly constrained using low-frequency data, then measurement of the spectral steepening readily points to the amplitude of the SZ effect, which in turn can be used for an independent derivation of the Mach number given some estimates for the pre-shock density and temperature. We further explore this issue in Sect. 4.

\subsubsection{The Toothbrush relic in $1 \mathrm{RXS} J 0603.3+4214$}

The famous Toothbrush relic is the other example where a spectral steepening has been observed at high frequencies (Stroe et al. 2016) and the modeling of its SZ contamination is almost identical in procedure as in the Sausage relic. The $\sim 2 \mathrm{Mpc}$ linear shape of the relic is peculiar, but following Brüggen et al. (2012) we assume that it is also caused by a merger shock. The Mach number determined from radio and X-ray data are similarly discrepant as in Sausage. van Weeren et al. (2012) obtained the radio synchrotron spectrum and determined a high $\mathcal{M}=4.6$ based on DSA theory, whereas Suzaku X-ray analysis by Itahana et al. (2015) provide a value of only $\mathcal{M}=1.5$. The cluster $X$-ray temperature of $7.3 \mathrm{keV}$ quoted by Itahana et al. (2015) gives an X-ray based mass of roughly $M_{500}^{\mathrm{X}-\text { ray }}=6.6 \times 10^{14} M_{\odot}$, and a
Planck derived $Y_{500}$ value of $(4.1 \pm 0.1) \times 10^{-3} \operatorname{arcmin}^{2}$ suggests roughly $70 \%$ more mass for the pressure calculation. The assumed relic geometry is $L L S=1.9 \mathrm{Mpc}$ and $r_{\text {shock }}=1.08 \mathrm{Mpc}$, following van Weeren et al. (2012).

The spectra for the Toothbrush relic, shown in the bottom panel of Fig. 10, provides a much better fit to the highfrequency measurements of Stroe et al. (2016), as compared to the Sausage relic. The contamination values are very similar, and the modified spectra support a high value of Mach number $(\mathcal{M} \sim 3.5)$ than those suggested from X-ray measurements (although, as in the Sausage spectrum, the low-frequency data cannot be fit satisfactorily by a single power law spectrum).

\subsubsection{The NW relic in El Gordo (ACT-CL J0102-4915)}

The two El Gordo cluster relics (Lindner et al. 2014) present an exciting case for computing the SZ flux contamination at $\mathrm{cm}$ wavelengths. At $z=0.87$ this cluster hosts both the highest redshift radio relics and radio halo known to date, and we already discussed the case for its radio halo in Sect. 2.4. Here we present the SZ flux contamination estimation for its more prominent of the two relics, the NW relic. Its highest frequency measurement is at $2.1 \mathrm{GHz}$ with ATCA, and some evidence for spectral steepening is found from the inter-bandpass measurement $\left(\alpha_{0.6}^{2.1}=1.2 \pm 0.1\right.$ and $\alpha_{1.6}^{2.6}=2.0 \pm 0.2$; see Lindner et al. 2014). We check whether the SZ flux contamination can be effective already at this very low frequency.

For the El Gordo NW relic our lognormal emissivity model provides an excellent fit to the published radio synchrotron brightness profile (Fig. 2), supporting an edge-on relic. The GNFW-model based global SZ prediction for the cluster, with X-ray derived mass $M_{500}=11.7 \times 10^{14} M_{\odot}$ (Menanteau et al. 2012), is consistent with the ACT and SPT published values (Marriage et al. 2011; Williamson et al. 2011), so no additional SZ flux correction is needed. There is only a radio (spectral index based) measurement of the Mach number, $\mathcal{M}=2.5_{-0.3}^{+0.7}$ (Lindner et al. 2014), which we adopt here. The results do not support any significant flux contamination at the published ATCA band. At $3 \mathrm{GHz}$ the flux contamination is less than $1 \%$, and at $5 \mathrm{GHz}$ the flux contamination is $3 \%$. Thereafter it increases rapidly; at $15 \mathrm{GHz}$ only half of the true synchrotron flux can be observed, and at $30 \mathrm{GHz}$ the synchrotron flux is completely swamped by the negative SZ signal (Table 1). This shows that measurements along the line of Sausage relic are completely erroneous for the $\mathrm{El}$ Gordo cluster already at $10 \mathrm{GHz}$ if the SZ effect is not taken into account, even though the weak spectral steepening suggested from the current low-frequency data appears to be safe from SZ contamination.

\subsubsection{A sample of double (edge-on) relics}

We present SZ contamination result predictions for a nearcomplete sample of double relic clusters, as compiled by de Gasperin et al. (2014) and Vazza et al. (2015). We can reasonably assume all of these relics are viewed edge-on. Reported radio spectral indices are used to derive the shock Mach numbers. To compute the SZ fluxes, first cluster masses are derived from the soft-band $X$-ray luminosities given in the above publications, and then the resulting SZ signals are corrected by the $Y_{500}$ values extracted uniformly from the $y$-maps derived from Planck data (see Sect. 3.2.1). All other relevant information, such as the relic LLS, cluster-centric distances, and the $1.4 \mathrm{GHz}$ radio power, are available for this compilation. 


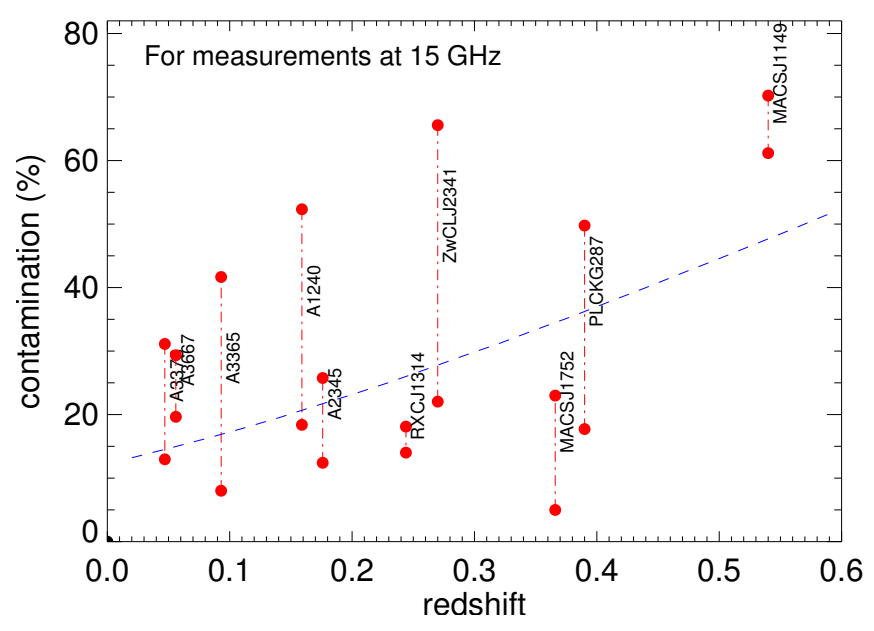

Fig. 11. Contamination predictions for a set of double relics in ten galaxy clusters, for whom the edge-on geometry can be applied with reasonable confidence. The relic dimensions and flux measurements are compiled by de Gasperin et al. (2014) and Vazza et al. (2015); the SZ predictions are calibrated against actual $Y_{500}$ values derived from Planck data. The blue dashed line is the mean contamination prediction from our modeling (see Sect. 3.1.1), using the mean cluster mass and shock parameters for this sample. Red dot-dashed lines connect the relic pairs for easier identification.

In Fig. 11 the contamination predictions at $15 \mathrm{GHz}$ are shown for this double relic sample. The mean contamination at this frequency is in the range $10-50 \%$, increasing with redshift, which we show with the blue dashed line. This curve corresponds to the mean mass and Mach number $\left(\bar{M}_{500} \sim 5.5 \times\right.$ $10^{14} M_{\odot}, \bar{M} \sim 2.5$ ) for the sample. The actual cluster-specific values exhibit a large scatter around this mean curve and, furthermore, we see that the SZ contamination for two relics in the same cluster can differ by a factor of several. This is caused by both the difference in their observed radio powers and the cluster-centric distances, the latter resulting in very different ambient (pre-shock) pressures in our model. Combined with the difficulty of accurately determining the shock Mach number from radio data, this scatter in the SZ contamination can make it very uncertain to use high-frequency relic measurements to constrain the general properties of shock acceleration or electron aging.

\subsubsection{Radio relic in $\mathrm{A} 2256$}

The radio relic in A2256 is the only case in which an attempt was made to estimate the SZ flux contamination (Trasatti et al. 2015). These authors presented $10 \mathrm{GHz}$ data from the Effelsberg single-dish receiver, and using a simple order-of-magnitude calculation, estimated the SZ flux contamination to be on the order of $25 \%$ at that frequency. However, Trasatti et al. argued that this contamination is an upper limit of the effect, and in any case, the concern of the paper was with the exceedingly flat synchrotron spectrum that is in tension with simple DSA-based stationary shock models. Given the challenges faced by the standard relic models to explain the A2256 radio emission (see e.g., Clarke \& Ensslin 2006; van Weeren et al. 2012; Trasatti et al. 2015), we do not attempt to explain the emission scenario, but rather use the a priori assumption that the emission is indeed a shock-induced radio relic, and make a realistic estimate for the SZ contamination based on our model.

The modeling of the A2256 synchrotron emission profile poses an additional problem with our adopted lognormal emissivity model. This profile cannot be fitted very well with the

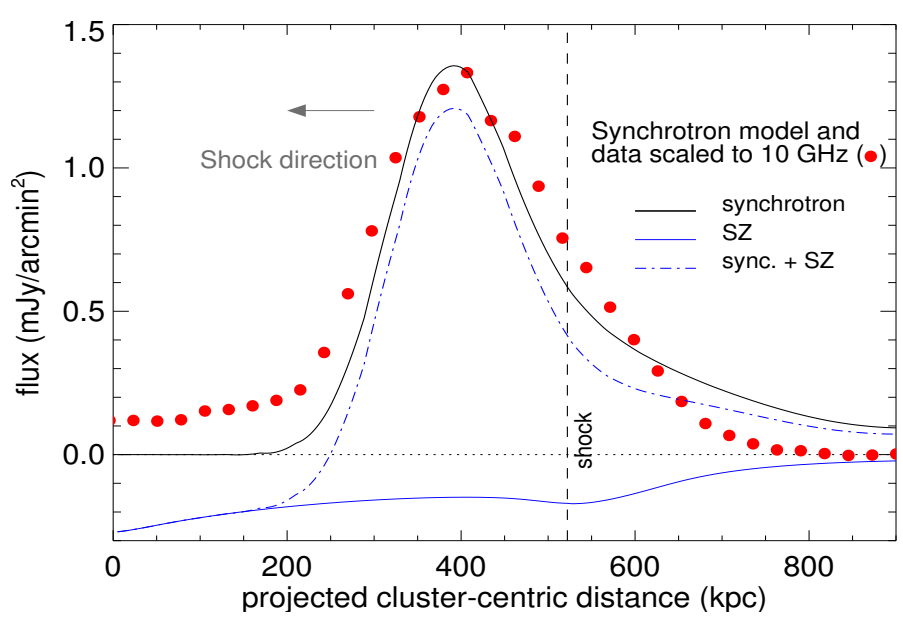

Fig. 12. Schematic model for the synchrotron and SZ flux profiles for the A2256 relic at $10 \mathrm{GHz}$. The geometry assumes a $\mathcal{M}=2$ shock propagating inward at roughly $500 \mathrm{kpc}$ distance from the cluster center, and viewed at an angle of $40^{\circ}$. The black solid line is the synchrotron flux profile, and the blue dot-dashed line is the SZ profile. The red points are the measured $1.4 \mathrm{GHz}$ flux density values from Clarke \& Ensslin (2006), scaled to the considered frequency.

standard outwardly propagating shock that we have considered so far. In addition there is the strong likelihood that the relic is viewed at a large angle. We find an acceptable fit (Fig. 12) by allowing the shock to move inward, when the relic is viewed at approximately $40^{\circ}$. This scenario is almost identical to that presented by Clarke \& Ensslin (2006) in their Fig. 11a. The peak of the relic emission is at a projected distance of $400 \mathrm{kpc}$ from the cluster center (van Weeren et al. 2012), which means the preshock material is actually $520 \mathrm{kpc}$ away and, hence, the ambient SZ signal lower by roughly factor 1.6. In Fig. 12 we show the expected fluxes at $10 \mathrm{GHz}$, where the synchrotron is scaled down by a spectral slope $\alpha_{\text {tot }}=0.81$ (van Weeren et al. 2012). The red data points correspond to the scaled-down version of the actual flux profile measured by Clarke \& Ensslin (2006). We assume the shock Mach number to be $\mathcal{M}=2$, for simplicity and for comparison with the estimate in Trasatti et al. (2015). One consequence of the large viewing angle and our assumption of a fullspherical shock for the SZ signal is that the synchrotron emission appears strongly shifted from the SZ shock feature, as is seen in Fig. 12. The relic falls into the more "uniform" SZ decrement area, and as such interferometric measurements might be able to recover almost all the lost flux, but the published $5-10 \mathrm{GHz}$ measurements of Trasatti et al. (2015) are made with a singledish (Effelsberg $100 \mathrm{~m}$ ) and should bear the full effect of the large-scale SZ decrement.

After adjusting the cluster pressure profile to the Planck derived $Y_{500}$ value (equivalent mass $M_{500}=7.9 \times 10^{14} M_{\odot}$ ), and assuming the shock and relic geometry as described above, we predict a radio flux contamination of roughly $1 \%$ at $3 \mathrm{GHz}$ and $3 \%$ at $5 \mathrm{GHz}$. These numbers are smaller than the current measurement uncertainties. At $10 \mathrm{GHz}$ the flux contamination is more significant at roughly $28 \%$. This also turns out to be nearly identical to the rough estimate made in Trasatti et al. (2015), calculating an SZ flux of $\sim-20 \mathrm{mJy}$ compared to the measured radio flux $61.7 \mathrm{mJy}$ at $10.45 \mathrm{GHz}$. Nevertheless, contrary to the suggestion given in Trasatti et al. (2015), our new estimates should not be considered an upper limit for the SZ effect. Several factors can result in a stronger SZ contamination, such as closer distance to the cluster center, a higher shock Mach number, or integrating over some negative signal in a single-dish observation. From our 
current modeling and idealized flux measurement setup, we predict that the synchrotron signal will be completely dwarfed by the SZ decrement above $20 \mathrm{GHz}$ for the A2256 relic.

The contamination values for the A2256 relic are much stronger than the other low $z$ relics we have considered so far (see Table 1). One reason is the low radio luminosity for this relic compared to both Sausage and Toothbrush relics given their respective cluster masses; the total $1.4 \mathrm{GHz}$ rest-frame power for A2256 is $P_{1.4}=3.6 \times 10^{24} \mathrm{~W} / \mathrm{Hz}$ (Clarke \& Ensslin 2006), about factor 2.5 lower than the mean relic power scaling of de Gasperin et al. (2014), whereas both Sausage and Toothbrush relics are highly overluminous. It is also possible that we overestimate the SZ owing to projection from our full-spherical shock model, when the relic is viewed at a large angle. However, in such a case the clusterwide SZ signal will be the main contributor for the flux decrement (Fig. 12), as the relic appears closer to the cluster center. Similar considerations also affect the Coma relic measurement as discussed below.

\subsubsection{The Coma relic}

The Coma cluster hosts the other well-known radio relic in the local universe, and in Erler et al. (2015) we presented the first measurement of a radio relic shock Mach number through the SZ signal variation. The results in Erler et al. (2015) were based on a cylindrical shock model, and for consistency with the other results in this paper we fit the Planck derived Coma SZ profile with a spherical shock model. We also use the latest 2015 data release of Planck for the $y$-map extraction. The result is a shock with $\mathcal{M}=2.2 \pm 0.3$, which is consistent with the Erler et al. (2015) result. The shock radius is fixed at $2.1 \mathrm{Mpc}$ from Coma cluster center $\left(75^{\prime}\right)$. Unlike A2256, the current multiwavelength data clearly supports an outward-moving shock for the Coma relic (see discussion in Erler et al. 2015), even though several earlier works argued for a different scenario (e.g., Feretti \& Neumann 2006; Brown \& Rudnick 2011). Modeling the relic synchrotron emission with our lognormal model also lends support to an outward moving shock, where the relic is seen at an angle of roughly $30^{\circ}$ (see Fig. 3). Interestingly, this is very close to the viewing angle of $\sim 37^{\circ}$ originally proposed by Enßlin et al. (1998).

We use the total relic flux and spectral index from Feretti et al. (2012) and the clusterwide GNFW pressure fit result obtained in Erler et al. (2015). The contamination estimates are listed in Table 1. Up to $10 \mathrm{GHz}$ the effect of SZ contamination is small, but at $20 \mathrm{GHz}$ the contamination is approximately $40 \%$, and at $30 \mathrm{GHz} 80 \%$. Similar to A2256, these contamination values are also high for a local universe cluster, likely for the same reasons discussed earlier (in particular, the radio luminosity is an order of magnitude lower than the mean relation of de Gasperin et al. 2014). Present and future single-dish observations of the Coma relic should exercise particular care in modeling its total flux above $10 \mathrm{GHz}$.

\section{Discussion}

We have shown that the SZ flux contamination is something that cannot be ignored when interpreting the current and future $\mathrm{cm}$ wavelength data for cluster radio relics, but can we learn something new from this? In this section we speculate on the implications of the thermal vs nonthermal measurements at $\mathrm{cm}$ wavelengths, and derive a simple analytical expression for the synchrotron-to-SZ flux ratio. To demonstrate the practical advantages that might be gained from measuring this ratio, we propose a possible method to constrain the relic magnetic field or the shock acceleration efficiency.

\subsection{Thermal modification of the nonthermal signal}

A significant SZ decrement at $\mathrm{cm}$ wavelengths is an unavoidable result of the widely assumed shock-relic connection. The only way to avoid SZ contamination is to assume that the gas pressure distribution across the relic location is much smoother than the radio synchrotron emission (so that the SZ signal can be almost entirely filtered out by radio-interferometric observations). However, this would force a complete reconsideration of the shock scenario necessary for the electron acceleration and the emergence of relics. The uncertainties in the actual amplitude of the SZ decrement only depends on "simple" physical ingredients, such as the unknown gas pressure, Mach number, exact profile of the shock, and geometry of the system. These factors make our estimate uncertain within a factor $\sim 2$ in most cases. Given this uncertainty, the fact that the estimated SZ decrement is on the order of the observed steepening of high frequency radio spectra of relics can be regarded as one of the best evidence so far that relics are actually connected to shocks, as the two signals (synchrotron emission and SZ decrement) are nearly equally sampled by radio observations.

Once we assume that the observed spectral steepening is solely due to the SZ effect, then the spectral curvature provides a straightforward way to measure the SZ effect itself, and model the shock Mach number (if the spectrum at the low-frequency end is modeled by a single power law). This "derived" SZ amplitude can be compared against independent SZ measurements at millimeter/submillimeter wavelengths, or from combining X-ray results. If a spectral steepening is found even after correcting for the SZ flux bias (as might be the case in the NW relic of El Gordo), then a case can be made for nonstandard scenarios in the shock acceleration model. An additional test can be for the shock Mach number itself. The current thermal (X-ray) and nonthermal (synchrotron) Mach number estimates are often in tension, whereas we assumed the same shock Mach number for SZ and synchrotron flux modeling throughout. If observations of the low-frequency synchrotron spectral slope and the highfrequency spectral steepening can both be explained by the same Mach number, then that is a strong indicator for the true Mach number of the underlying merger shock.

\subsection{Analytical model for the synchrotron-to-SZ flux ratio}

We now wish to derive a simple analytical expression for the synchrotron-to-SZ flux ratio that can provide a quantitative measure for the spectral steepening at $\mathrm{cm}$ wavelengths. This requires connecting the properties of the cosmic ray electrons to those in the thermal gas, which brings in heavily model-dependent assumptions. For our illustration, we use the DSA-based model for relic radio synchrotron emission by Hoeft \& Brüggen (2007) in the simplified form, as given by Vazza et al. (2015),

$$
\begin{aligned}
P_{v}^{\text {sync. }} \approx 3.5 & \times 10^{26} \mathrm{~W} / \mathrm{Hz}\left(\frac{L^{2}}{1 \mathrm{Mpc}^{2}}\right)\left(\frac{n_{\mathrm{u}}}{10^{-4} \mathrm{~cm}^{-3}}\right)\left(\frac{T_{\mathrm{u}}}{1 \mathrm{keV}}\right)^{3 / 2} \\
& \times\left(\frac{v}{1.4 \mathrm{GHz}}\right)^{-\delta / 2} \frac{B_{\text {relic }}^{1+\delta / 2}}{B_{\mathrm{CMB}}^{2}+B_{\text {relic }}^{2}}\left(\frac{\xi_{\mathrm{e} / \mathrm{p}}}{0.05}\right) \eta(\mathcal{M}) .
\end{aligned}
$$

In this formulation of the total relic power, $L$ is the observed relic length (hence approximating the relic surface area as $A \approx L^{2}$ ), $n_{\mathrm{u}}$ and $T_{\mathrm{u}}$ are the upstream (pre-shock) electron density and temperature, $\xi_{\mathrm{e} / \mathrm{p}}$ is the electron-to-proton ratio, and $\eta(\mathcal{M})$ is the shock 
acceleration efficiency. The spectral index and the magnetic field carry Mach number dependence as well, through the electron momentum power law index $\delta$ (cf. Eq. (8)). The parameter $B_{\text {relic }}$ is the relic magnetic field in $\mu \mathrm{G}$, and $B_{\mathrm{CMB}}=3.24(1+z)^{2} \mu \mathrm{G}$.

The simplified expression of Eq. (11) can be expected to match real observations only within an order of magnitude; the main theoretical uncertainty comes from the electron acceleration efficiency, given by $\xi_{\mathrm{e} / \mathrm{p}} \cdot \eta(\mathcal{M})$. We described its behavior in the DSA-based model of Hoeft \& Brüggen (2007) in Sect. 3.1.3, referring to the fitting formula in Kang et al. (2007) for the case without a pre-existing CRe component when $\mathcal{M}>2$. However, there is much theoretical uncertainty in this term, and as Kang et al. (2012) have shown, several combinations of pre-shock cosmic rays and injection efficiency can explain the same observed relic emission. The fitting formula of Kang et al. (2007) can be approximated near $\mathcal{M} \sim 3$ by $\eta(\mathcal{M}) \approx 0.004 \mathcal{M}^{3}$, however, using this approximation the synchrotron power for the Sausage relic is overpredicted by a factor of 13 (against the measured value of $P_{1.4}=1.5 \times 10^{25} \mathrm{~W} / \mathrm{Hz}$; de Gasperin et al. 2014). In reality this discrepancy can be due to several other unknowns, such as the upstream density, $\mathrm{CR}$ e/p ratio, or the relic magnetic field, but we make the assumption that this discrepancy comes mainly from the efficiency term $\eta(\mathcal{M})$, and fix its normalization as $\eta(\mathcal{M}) \approx 3 \times 10^{-4} \mathcal{M}^{3}$ near $\mathcal{M} \sim 3$. With this approximation the Eq. (11) matches real observations more closely.

After this "recalibration" of the radio synchrotron power, we can write the total monochromatic flux observed from a radio relic, assuming standard isotropic flux distribution, as

$$
\begin{aligned}
S_{v}^{\text {sync. }}= & (1+z)^{-(\delta / 2)} P_{v}^{\text {sync. }} / 4 \pi D_{\mathrm{L}}^{2} \\
\approx & 24 \mathrm{mJy}\left(\frac{\mathcal{M}}{3}\right)^{3}\left(\frac{\xi_{\mathrm{e} / \mathrm{p}}}{0.05}\right)\left(\frac{L^{2}}{1 \mathrm{Mpc}^{2}}\right) \frac{B_{\text {relic }}^{1+\delta / 2}}{B_{\mathrm{CMB}}^{2}+B_{\text {relic }}^{2}} \\
& \times\left(\frac{n_{\mathrm{u}}}{10^{-4} \mathrm{~cm}^{-3}}\right)\left(\frac{T_{\mathrm{u}}}{1 \mathrm{keV}}\right)^{3 / 2}\left(\frac{D_{\mathrm{L}}}{10^{3} \mathrm{Mpc}}\right)^{-2} \\
& \times(1+z)^{-\delta / 2}\left(\frac{v}{1.4 \mathrm{GHz}}\right)^{-\delta / 2} .
\end{aligned}
$$

In the above flux formula $(1+z)^{-\delta / 2}$ represents the $K$-correction due to the falling synchrotron spectrum with index $\alpha_{\text {tot }}=\delta / 2$, ignoring an additional $(1+z)$ term for the bandwidth. $D_{\mathrm{L}}$ is the luminosity distance and in the second step we assumed a cluster at $z=0.2$ such that $D_{\mathrm{L}} \approx 10^{3} \mathrm{Mpc}$. Henceforth we are also using the approximation $\eta(\mathcal{M}) \approx 3 \times 10^{-4} \mathcal{M}^{3}$, which greatly simplifies the Mach dependence in our formulas but is only valid for a narrow range around $\mathcal{M} \sim 3$.

The SZ "flux" is not isotropic, it depends on the line-of-sight projection of the pressure and, therefore, relies on the shock geometry at the location of the relic. For an edge-on relic we can consider only the shock-boosted pressure at the relic location, ignoring the large-scale SZ signal gradient. To simplify the projection we also consider plane-parallel geometry, ignoring curvature. The line-of-sight depth is the same as the relic length in the plane of the sky, $L$. Then from Eq. (4) the Compton- $y$ parameter just behind the shock front would be

$y \approx 3.6 \times 10^{-6}\left(\frac{n_{\mathrm{u}} T_{\mathrm{u}}}{10^{-4} \mathrm{keV} \mathrm{cm}^{-3}}\right)\left(\frac{L}{1 \mathrm{Mpc}}\right)\left(\frac{\mathcal{M}}{3}\right)^{2}$.

In keeping with the synchrotron modeling we have taken the relic LLS to be $1 \mathrm{Mpc}$ and shock Mach number $\sim 3$. We also approximated the shock-boosted pressure in Eq. (6) as $P_{\mathrm{d}} / P_{\mathrm{u}} \approx$ $\mathcal{M}^{2}$. This $y$-value translates into a brightness (or RayleighJeans) temperature, at frequencies $\lesssim 30 \mathrm{GHz}$, of roughly $\Delta T_{\mathrm{RJ}} \approx$ $-20 \mu \mathrm{K}$, with the same shock parameters as in Eq. (13) above.
To compute the total SZ flux we need to integrate $y$ over the visible relic area. We take the mean relic width to be $W \approx$ $100 \mathrm{kpc}$, such that the angular size of the relic is $\Omega_{\text {relic }} \approx$ $L \mathcal{W} / D_{\mathrm{A}}^{2}$ steradians, where $D_{\mathrm{A}}$ is the angular diameter distance. Therefore, applying Eq. (3) we obtain the following expression for the total SZ flux decrement,

$$
\begin{aligned}
S_{v}^{\mathrm{SZ}} \approx-0.26 \mu \mathrm{Jy} & \left(\frac{D_{\mathrm{A}}}{700 \mathrm{Mpc}}\right)^{-2}\left(\frac{L}{1 \mathrm{Mpc}}\right)^{2}\left(\frac{W}{100 \mathrm{kpc}}\right) \\
& \times\left(\frac{n_{\mathrm{u}} T_{\mathrm{u}}}{10^{-4} \mathrm{keV} \mathrm{cm}^{-3}}\right)\left(\frac{\mathcal{M}}{3}\right)^{2}\left(\frac{v}{1.4 \mathrm{GHz}}\right)^{2} .
\end{aligned}
$$

The SZ flux is proportional to $v_{\mathrm{obs}}^{2}$ in the RJ-limit, and we have taken $D_{\mathrm{A}}=700 \mathrm{Mpc}$ for a $z \sim 0.2$ cluster in accordance with the derivation above. We see that the SZ signal has the same $L^{2}$ dependence as in the synchrotron with an additional width term $\mathcal{W}$ that needs to be determined observationally.

The synchrotron-to-SZ flux ratio, as a function of frequency, is therefore

$$
\begin{aligned}
\frac{S_{v}^{\text {sync. }}}{S_{v}^{\mathrm{SZ}}} \approx & -9 \times 10^{4}\left(\frac{\xi_{\mathrm{e} / \mathrm{p}}}{0.05}\right)\left(\frac{\mathcal{M}}{3}\right)\left(\frac{T_{\mathrm{u}}}{1 \mathrm{keV}}\right)^{1 / 2}\left(\frac{\mathcal{W}}{100 \mathrm{kpc}}\right)^{-1} \\
& \times(1+z)^{-(4+\delta / 2)} \frac{B_{\text {relic }}^{1+\delta / 2}}{B_{\mathrm{CMB}}^{2}+B_{\text {relic }}^{2}}\left(\frac{v}{1.4 \mathrm{GHz}}\right)^{-(2+\delta / 2)}
\end{aligned}
$$

The gas density and relic LLS dependence is absent from the flux ratio; its primary dependence is on the uncertain particle acceleration efficiency term and the e/p ratio, and to a lesser extent on the shock geometry and the relic magnetic field. With the simplifying assumption $\eta(\mathcal{M}) \approx 3 \times 10^{-4} \mathcal{M}^{3}$, the Mach dependence has reduced to a simple linear scaling. The distances cancel out yielding $D_{\mathrm{A}}^{2} / D_{\mathrm{L}}^{2}=(1+z)^{-4}$, with additional $z$-dependence from the synchrotron $K$-correction.

\subsection{Magnetic field from the synchrotron-to-SZ flux ratio}

As one sees from Eq. (15), the flux ratio between the SZ and synchrotron signals can be used to make an estimation for the relic magnetic field. This requires several assumptions that are as-yet untested, for example, the CRe injection spectrum and acceleration efficiency at low Mach numbers. However, we wish to emphasize that once we make these assumptions, which can be motivated by current theory and observations, then the SZto-synchrotron flux ratio at $\mathrm{GHz}$ frequencies can be a particularly easy-to-measure tool for putting constraints on magnetic fields (or the shock acceleration efficiency). The direct observational method for constraining ICM magnetic fields, through the measurement of Faraday rotation angles of background polarized radio sources, have yielded results for only a handful of cases (e.g., Murgia et al. 2004; Bonafede et al. 2013). Indirect methods like measuring the inverse-Compton emission in hard X-rays so far could only yield upper limits (e.g., Bartels et al. 2015). Connecting the thermal signature of the shock with its nonthermal synchrotron emission can provide another indirect yet independent method.

The parameter dependence in Eq. (15) for the flux ratio can be made clearer if we take the strong field limit, $B_{\text {relic }} \gg B_{\mathrm{CMB}}$, such that the term containing the magnetic field becomes approximately $B_{\text {relic }}^{\delta / 2-1}$. Then from Eq. (15) we get for the magnetic field strength

$B_{\text {relic }} \propto\left[-\left(\frac{S_{v}^{\text {sync. }}}{S_{v}^{\mathrm{SZ}}}\right) \frac{W v_{\mathrm{obs}}^{2+\delta / 2}}{\mathcal{M} \xi_{\mathrm{e} / \mathrm{p}} T_{\mathrm{u}}^{1 / 2}}\right]^{2 /(\delta-2)}$ (for $B_{\text {relic }} \gg B_{\mathrm{CMB}}$ ). 
The flux ratio term in parenthesis is just the inverse of contamination fraction, $C(\%) \equiv 100 \times\left(S_{v}^{\mathrm{SZ}} / S_{v}^{\text {sync. }}\right)$, which is easy to determine observationally. With the current broadband receivers a single low-frequency ( $\lesssim 1 \mathrm{GHz}$ ) measurement provides the true synchrotron flux as well as the spectral slope and, hence, the value of $\delta$, provided the low-frequency relic spectrum is close enough to a power law. The Mach number is derived from the spectral slope as $\delta=2\left(\mathcal{M}^{2}+1\right) /\left(\mathcal{M}^{2}-1\right)$, or alternatively, independent X-ray or SZ measurements can provide the value of $\mathcal{M}$. The electron-to-proton ratio can be considered fixed at some universal value of roughly $5 \%$. Then one single high-frequency measurement of the radio flux yields the departure from the synchrotron power law and, hence, the SZ-to-synchrotron flux ratio.

This procedure basically uses the SZ signal to constrain the pre-shock gas properties in a DSA-based synchrotron model (Eq. (11)). We emphasize that the same task can also be done with X-ray spectral imaging data, although obtaining upstream temperatures from X-ray data can be difficult, particularly at high redshifts. The temperature dependence in Eq. (16) is weak, for massive clusters near $1 \mathrm{Mpc}$ radius we generally have $T_{\mathrm{u}} \sim$ 2-3 keV, so the uncertainty from the temperature term is small. We eliminated the density dependence, which can vary by a much wider margin than temperature at the cluster outskirts (see discussion in Bonafede et al. 2013 for the Coma relic). The relic width $\mathcal{W}$ is determined readily from high-resolution radio observation. Therefore, if the Mach dependence of particle acceleration efficiency is known from a model or can be constrained using independent observations, a constraint on the relic magnetic field follows.

As a demonstration, we derive the magnetic field strength for the Sausage relic, from Eq. (15), to show that it provides reasonable values despite the assumptions we have made. From our modeling of the SZ contamination in Sausage radio measurements (Sect. 3.2.1), we get roughly 3\% contamination for $\mathcal{M}=3$ for an observation at $10 \mathrm{GHz}$, i.e., $S_{v}^{\mathrm{SZ}} / S_{v}^{\text {sync. }} \approx-0.03$. The particle energy index is $\delta=2.5$ for this choice of Mach number. The measurement by van Weeren et al. (2010) show a mean half-power width of the relic emission at $0.6 \mathrm{GHz}$ roughly $\mathcal{W} \approx 70 \mathrm{kpc}$, and we take the unshocked (upstream) temperature $T_{\mathrm{u}}=2 \mathrm{keV}$. Then using our approximation for the acceleration efficiency $\eta(\mathcal{M})$ at $\mathcal{M} \sim 3$, we get a value for the Sausage relic magnetic field strength of roughly $B_{\text {relic }} \approx 2.5 \mu \mathrm{G}$ (or $3 \mu \mathrm{G}$ for relic width $\mathcal{W}=100 \mathrm{kpc})$. This is somewhat lower than the equipartition magnetic field at this cluster's redshift, and fully consistent with the best-fit estimate of van Weeren et al. (2010).

\section{Summary and conclusions}

Motivated by some recent observations of galaxy cluster radio relics at frequencies above $10 \mathrm{GHz}$, we investigated how the Sunyaev-Zel'dovich (SZ) effect signal might affect these observations if the radio relics are manifestations of cluster merger shocks. This problem has not been addressed in detail so far, and we developed a semianalytical model for quantitative prediction. The primary argument came from the fact that shocks at radio relics boost the ambient pressure of the intracluster medium locally by roughly an order of magnitude (scaling as $\sim \mathcal{M}^{2}$ ). Thus even though relics are located in the cluster outskirts where ambient pressure is low, the local shock boost generates a negative SZ signal that can be significant at $\mathrm{cm}$ wavelengths. Measurements made from interferometers are not fully immune to the flux contamination, as the change in the SZ signal is localized roughly within the same angular scales as the synchrotron emission. The main steps for our modeling were as follows:

- We assumed a spherical shock model for creating the pressure boost at the shock front, which is added on top of a clusterwide spherical pressure that follows the universal GNFW profile, scaled to the cluster mass and redshift. These were then projected together to obtain the SZ decrement at the required frequency.

- We modeled the radio synchrotron emission semianalytically with a lognormal emissivity profile, which we matched to the published low-frequency radio data. The shape, length, and total power of the synchrotron emission were fitted on caseby-case basis for individual cluster predictions. In addition, we used a fiducial relic geometry and an empirical scaling law between the total relic power and cluster mass to compute flux contamination trends against redshift and mass.

- We performed realistic simulations to mimic observations made by interferometers and found that interferometric imaging also reports flux loss due to the SZ effect. Moreover, most interferometers are affected by a lack of total power measurement due to the large angular sizes of radio relics. The actual interferometric measurements can be higher or lower than the "idealized" contamination predictions that we reported, depending on the array configuration, deconvolution methods, etc., but are roughy of similar magnitude.

Our results can be divided in two parts: first is a word of caution for observers dealing with current and future radio relic data at $\mathrm{cm}$ wavelengths, and second is a proposal for future theoretical works to model the SZ-to-synchrotron flux ratio in greater detail.

\section{Caution for observers}

SZ flux contamination cannot be neglected for radio relic observations at $v_{\mathrm{obs}} \gtrsim 10 \mathrm{GHz}$ in almost all of the cases. We compared the most recent data for the Sausage and Toothbrush relics up to $30 \mathrm{GHz}$ and found that $\mathrm{SZ}$ contamination can explain the observed spectral steepening to a large extent. At $10 \mathrm{GHz}$ their flux contamination is roughly $10 \%$, comparable to the current measurement uncertainties, but at $30 \mathrm{GHz}$ the contamination reaches $50 \%$ level, i.e., only half of the original synchrotron flux is observed.

We presented estimates for a set of double relics and found their contamination to scatter between $10-70 \%$ level. We also investigated two famous radio relics in the local universe: in A2256 and Coma clusters. Their synchrotron profile modeling indicated that these single relics might be viewed at large angles, especially in A2256. The SZ flux contamination is also large: for A2256 it is roughly $30 \%$ at $10 \mathrm{GHz}$ and close to $100 \%$ at $20 \mathrm{GHz}$, whereas for Coma it is lower, about $6 \%$ and $40 \%$ at these frequencies. Such high contaminations can be a concern for single-dish observations of local universe radio relics.

Because of the redshift independence of the SZ brightness, flux contamination goes up rapidly with redshift, and we found roughly an order of magnitude increase between redshifts 0 and 1. In addition, an empirical mass scaling of the relic synchrotron power suggested a higher contamination in low mass systems. We applied our model to the highest redshift radio relic known, the NW relic in the El Gordo cluster $(z=0.87)$, and found this relic to have roughly $50 \%$ flux contamination at $15 \mathrm{GHz}$ and its synchrotron flux would be completely eclipsed by the negative SZ signal near $30 \mathrm{GHz}$. This redshift trend is particularly relevant for the large number of intermediate- to high- $z$ radio relics 
that are expected to be discovered by upcoming surveys with ASKAP, MeerKAT, and SKA.

Thus it might be premature to consider nonstandard particle acceleration scenarios or other physical processes are at play at high frequencies based solely on a direct measurement of spectral steepening for radio relics. There can be some degree of intrinsic steepening occurring still, for example, as hinted from the Sausage or the El Gordo NW relic data. However, the amplitude required by the nonstandard scenarios can only be constrained in a meaningful way once the impact of the SZ effect has been robustly constrained in conjunction with instrument-specific imaging techniques.

\section{Challenge for theorists}

Far from being a simple "nuisance" for radio observations, the SZ decrement at $\mathrm{cm}$ wavelengths offers probably the best evidence for the relic-shock connection. We outlined some possible advantages that might be gained from the measurement, and also a more precise modeling, of the synchrotron-to-SZ flux ratio. For example, if the spectral slope of the synchrotron spectrum at low frequencies can be robustly constrained, then the observed amplitude of the steepening can inform the Mach number of the shock in a way that is complementary to the standard radio estimate using the spectral slope from the synchrotron spectrum (Sect. 4.1).

We provided a simple analytical approximation for the synchrotron-to-SZ flux ratio (Sect. 4.2). This step required connecting the number (and energy) of the cosmic ray electrons to that of the thermal electrons present in the intracluster medium and is theoretically uncertain. We assumed a DSA-based model for the synchrotron emission that includes several unknowns such as the shock acceleration efficiency at low Mach numbers or the cosmic ray e/p ratio. The resulting flux ratio depends weakly on the thermal gas temperature, is independent of its density, and can provide certain practical advantages for constraining theoretical models for particle acceleration.

We proposed that the synchrotron-to-SZ flux ratio can be a tool for measuring the relic magnetic fields in the cluster outskirts, or the shock acceleration efficiency if magnetic field is known (Sect. 4.3). Despite making use of several restrictive theoretical assumptions for the analytical approximation, we were able to get realistic magnetic field strengths when plugging-in flux contamination values obtained in the earlier part of our work. The estimate was roughly $3 \mu \mathrm{G}$ for the Sausage relic. Our suggestion is that through an accurate theoretical modeling of the synchrotron-to-SZ flux ratio, important advances can be made toward an understanding of the radio relic origin, and the $\mathrm{cm}$ wavelength window will be critical for testing these theoretical models.

Acknowledgements. We thank Torsten Enßlin, Marcus Brüggen, and Annalisa Bonafede for helpful discussions, Monica Trasatti for sharing unpublished Coma relic results, and Tracy Clarke for providing data for the A2256 relic. We thank the anonymous referee for a thorough reading of the manuscript and pointing out a mistake in Eq. (15). F.V. acknowledges support from the grant FOR1254 and VA876/3-1, J.E. support from the grant SFB956, and M.S. support from the Transregio Program TR33, all of the Deutsche Forschungsgemeinschaft (DFG).

\section{References}

Akamatsu, H., \& Kawahara, H. 2013, PASJ, 65, 16

Akamatsu, H., van Weeren, R. J., Ogrean, G. A., et al. 2015, A\&A, 582, A87
Arnaud, M., Pratt, G. W., Piffaretti, R., et al. 2010, A\&A, 517, A92 Bartels, R., Zandanel, F., \& Ando, S. 2015, A\&A, 582, A20

Birkinshaw, M. 1999, Phys. Rep., 310, 97

Blandford, R., \& Eichler, D. 1987, Phys. Rep., 154, 1

Bonafede, A., Vazza, F., Brüggen, M., et al. 2013, MNRAS, 433, 3208

Brown, S., \& Rudnick, L. 2011, MNRAS, 412, 2

Brüggen, M. 2013, MNRAS, 436, 294

Brüggen, M., van Weeren, R. J., \& Röttgering, H. J. A. 2012, MNRAS, 425, L76

Brunetti, G., Rudnick, L., Cassano, R., et al. 2013, A\&A, 558, A52

Bykov, A. M., Paerels, F. B. S., \& Petrosian, V. 2008, Space Sci. Rev., 134, 141

Clarke, T. E., \& Ensslin, T. A. 2006, AJ, 131, 2900

de Gasperin, F., van Weeren, R. J., Brüggen, M., et al. 2014, MNRAS, 444, 3130

Enßlin, T. A. 2002, A\&A, 396, L17

Enßlin, T. A., \& Kaiser, C. R. 2000, A\&A, 360, 417

Enßlin, T. A., Biermann, P. L., Klein, U., \& Kohle, S. 1998, A\&A, 332, 395

Erler, J., Basu, K., Trasatti, M., Klein, U., \& Bertoldi, F. 2015, MNRAS, 447, 2497

Feretti, L., \& Neumann, D. M. 2006, A\&A, 450, L21

Feretti, L., Giovannini, G., Govoni, F., \& Murgia, M. 2012, A\&ARv, 20, 54

Guo, X., Sironi, L., \& Narayan, R. 2014a, ApJ, 794, 153

Guo, X., Sironi, L., \& Narayan, R. 2014b, ApJ, 797, 47

Hoeft, M., \& Brüggen, M. 2007, MNRAS, 375, 77

Hong, S. E., Ryu, D., Kang, H., \& Cen, R. 2014, ApJ, 785, 133

Itahana, M., Takizawa, M., Akamatsu, H., et al. 2015, PASJ, 67, 11314

Jee, M. J., Dawson, W. A., Stroe, A., et al. 2016, ApJ, 817, 179

Kang, H., \& Ryu, D. 2013, ApJ, 764, 95

Kang, H., Ryu, D., Cen, R., \& Ostriker, J. P. 2007, ApJ, 669, 729

Kang, H., Ryu, D., \& Jones, T. W. 2012, ApJ, 756, 97

Kang, H., Petrosian, V., Ryu, D., \& Jones, T. W. 2014, ApJ, 788, 142

Kardashev, N. S. 1962, Soviet Ast., 6, 317

Lindner, R. R., Baker, A. J., Hughes, J. P., et al. 2014, ApJ, 786, 49

Marriage, T. A., Acquaviva, V., Ade, P. A. R., et al. 2011, ApJ, 737, 61

Matsukiyo, S., Ohira, Y., Yamazaki, R., \& Umeda, T. 2011, ApJ, 742, 47

Menanteau, F., Hughes, J. P., Sifón, C., et al. 2012, ApJ, 748, 7

Miniati, F. 2000, Ph.D. Thesis, University of Minnesota, USA

Miniati, F. 2007, J. Comput. Phys., 227, 776

Murgia, M., Govoni, F., Feretti, L., et al. 2004, A\&A, 424, 429

Nagai, D., Kravtsov, A. V., \& Vikhlinin, A. 2007, ApJ, 668, 1

Nuza, S. E., Hoeft, M., van Weeren, R. J., Gottlöber, S., \& Yepes, G. 2012, MNRAS, 420, 2006

Ogrean, G. A., Brüggen, M., Röttgering, H., et al. 2013, MNRAS, 429, 2617

Pfrommer, C., \& Enßlin, T. A. 2004, MNRAS, 352, 76

Pfrommer, C., Springel, V., Enßlin, T. A., \& Jubelgas, M. 2006, MNRAS, 367, 113

Pfrommer, C., Enßlin, T. A., \& Springel, V. 2008, MNRAS, 385, 1211

Pinzke, A., Oh, S. P., \& Pfrommer, C. 2013, MNRAS, 435, 1061

Planck Collaboration V. 2013, A\&A, 550, A131

Planck Collaboration X. 2013, A\&A, 554, A140

Planck Collaboration XVI. 2016, A\&A, in press,

DOI: $10.1051 / 0004-6361 / 201527101$

Rudd, D. H., \& Nagai, D. 2009, ApJ, 701, L16

Ryu, D., Kang, H., Hallman, E., \& Jones, T. W. 2003, ApJ, 593, 599

Sarazin, C. L. 2002, in Merging Processes in Galaxy Clusters, eds. L. Feretti,

I. M. Gioia, \& G. Giovannini, Astrophys. Space Sci. Lib., 272, 1

Sayers, J., Czakon, N. G., Mantz, A., et al. 2013, ApJ, 768, 177

Skillman, S. W., Xu, H., Hallman, E. J., et al. 2013, ApJ, 765, 21

Sommer, M. W., \& Basu, K. 2014, MNRAS, 437, 2163

Stroe, A., Rumsey, C., Harwood, J. J., et al. 2014, MNRAS, 441, L41

Stroe, A., Shimwell, T., Rumsey, C., et al. 2016, MNRAS, 455, 2402

Sunyaev, R. A., \& Zel'dovich, Y. B. 1972, Comments on Astrophysics and Space Physics, 4, 173

Sunyaev, R. A., \& Zel'dovich, Y. B. 1980, MNRAS, 190, 413

Taylor, G. B., Carilli, C. L., \& Perley, R. A. 1999, ASP Conf. Ser. 180, Synthesis Imaging in Radio Astronomy II

Thierbach, M., Klein, U., \& Wielebinski, R. 2003, A\&A, 397, 53

Trasatti, M., Akamatsu, H., Lovisari, L., et al. 2015, A\&A, 575, A45

van Weeren, R. J., Röttgering, H. J. A., Brüggen, M., \& Hoeft, M. 2010, Science, 330,347

van Weeren, R. J., Röttgering, H. J. A., Rafferty, D. A., et al. 2012, A\&A, 543, A43

van Weeren, R. J., Intema, H. T., Lal, D. V., et al. 2014a, ApJ, 786, L17

van Weeren, R. J., Intema, H. T., Lal, D. V., et al. 2014b, ApJ, 781, L32

Vazza, F., \& Brüggen, M. 2014, MNRAS, 437, 2291

Vazza, F., Brüggen, M., Gheller, C., \& Brunetti, G. 2012, MNRAS, 421, 3375

Vazza, F., Eckert, D., Brüggen, M., \& Huber, B. 2015, MNRAS, 451, 2198

Williamson, R., Benson, B. A., High, F. W., et al. 2011, ApJ, 738, 139 